\title{
Proteasome inhibition preserves longitudinal growth of denervated muscle and prevents neonatal neuromuscular contractures
}

\author{
Sia Nikolaou, ${ }^{1}$ Alyssa A.W. Cramer, ${ }^{2}$ Liangjun Hu, ${ }^{1}$ Qingnian Goh, ${ }^{1}$ Douglas P. Millay, ${ }^{2,3}$ \\ and Roger Cornwall1,3,4,5 \\ 'Division of Orthopaedic Surgery, and 'Division of Molecular Cardiovascular Biology, Cincinnati Children's Hospital \\ Medical Center, Cincinnati, Ohio, USA. ${ }^{3}$ Department of Pediatrics, University of Cincinnati College of Medicine, Cincinnati, \\ Ohio, USA. ${ }^{4}$ Division of Developmental Biology, Cincinnati Children's Hospital Medical Center, Cincinnati, Ohio, USA. \\ ${ }^{5}$ Department of Orthopaedic Surgery, University of Cincinnati College of Medicine, Cincinnati, Ohio, USA.
}

\begin{abstract}
Muscle contractures are a prominent and disabling feature of many neuromuscular disorders, including the $\mathbf{2}$ most common forms of childhood neurologic dysfunction: neonatal brachial plexus injury (NBPI) and cerebral palsy. There are currently no treatment strategies to directly alter the contracture pathology, as the pathogenesis of these contractures is unknown. We previously showed in a mouse model of NBPI that contractures result from impaired longitudinal muscle growth. Current presumed explanations for growth impairment in contractures focus on the dysregulation of muscle stem cells, which differentiate and fuse to existing myofibers during growth, as this process has classically been thought to control muscle growth during the neonatal period. Here, we demonstrate in a mouse model of NBPI that denervation does not prevent myonuclear accretion and that reduction in myonuclear number has no effect on functional muscle length or contracture development, providing definitive evidence that altered myonuclear accretion is not a driver of neuromuscular contractures. In contrast, we observed elevated levels of protein degradation in NBPI muscle, and we demonstrate that contractures can be pharmacologically prevented with the proteasome inhibitor bortezomib. These studies provide what we believe is the first strategy to prevent neuromuscular contractures by correcting the underlying deficit in longitudinal muscle growth.
\end{abstract}

Authorship note: DPM and RC contributed equally to this work.

Conflict of interest: The authors have declared that no conflict of interest exists

Copyright: (c) 2019, American Society for Clinical Investigation.

Submitted: March 1, 2019

Accepted: October 16, 2019

Published: December 5, 2019.

Reference information: JCI Insight. 2019;4(23):e128454.

https://doi.org/10.1172/jci.

insight.128454.

\section{Introduction}

Neonatal brachial plexus injury (NBPI) and cerebral palsy (CP) are the 2 most common causes of neuromuscular dysfunction in childhood, occurring in a combined 1 per 200 live births (1-4). Despite differing in the type of neurologic lesion (lower vs. upper motor neuron), both conditions lead to similar muscle contractures, which dramatically reduce joint range of motion and limit the functional use of limbs for ambulating, reaching, and other activities of daily living. Furthermore, the muscular contractures alter the physical forces on the developing skeleton, leading to progressive dysplasia and dislocation of joints (5-9). These contractures are the primary driver of the need for rehabilitative and surgical therapies, assistive devices, and accommodations for daily functioning $(10,11)$. However, no existing treatment strategies alter the actual contracture pathology, and instead can worsen function by further weakening already abnormal muscles (12-15). As a result, the contractures and their secondary skeletal consequences remain unchecked, leading to pain, loss of physical function, and heavy reliance on costly health care and supportive services. It is therefore imperative to gain a better understanding of contracture pathogenesis to develop novel strategies to prevent contractures.

We developed a mouse model of NBPI that causes contractures precisely mimicking the human phenotype in both NBPI and CP (16). With this model, we have made several important discoveries regarding the pathogenesis of contractures. First, it was unknown whether the tissue responsible for the clinical contracture phenotype was the muscle or the joint capsule, pathology of which commonly causes other forms of contractures (17). We found that the elbow flexion contractures in our NBPI model could be completely 
relieved by excision of the denervated elbow flexor muscles, leaving the joint capsule intact (16). This finding highlights that NBPI-induced contractures are primarily due to tightness of denervated muscle, rather than pathology of other tissues such as bone, capsule, or ligaments. The next question was whether the tightness of the denervated muscle is a direct effect of denervation or a result of the muscle's altered mechanical environment. Neuromuscular contractures have previously been assumed to be the result of mechanical imbalance, whereby stronger muscles pull a joint into a contracted position due to weakness of antagonists (10). We demonstrated that neonatal denervation of the elbow flexors causes elbow flexion contractures, but neonatal surgical excision of the triceps alone does not (18). This finding rules out muscle strength imbalance as a cause of contractures, and instead highlights a direct effect of denervation on muscle tightness. Finally, it was unknown whether the tightness of denervated muscles resulted from fibrosis (muscle too stiff) or impaired longitudinal growth (muscle too short). Fibrosis, or increased extracellular matrix collagen accumulation, has been postulated to contribute to contractures in both NBPI and CP, owing to findings on muscle biopsies after contractures have formed $(19,20)$. However, when we used our model to interrogate the role of fibrosis during contracture development, we found that fibrosis is not a causative mechanism (21). Indeed, fibrosis occurs after the onset of contractures and pharmacologic reduction of fibrosis after denervation does not prevent contractures (21). In contrast, we observed that within 4 weeks of NBPI, during the time frame of contracture development, denervated muscles exhibited elongated sarcomeres compared with contralateral control muscles, which was analyzed by controlling for joint angle and bone length (16, 18). This sarcomere elongation correlated with contracture severity, and indicates fewer sarcomeres in series in the whole muscle (22), or impaired longitudinal whole muscle growth. This finding that neuromuscular contractures are caused by muscle that has failed to grow normally in length as a direct result of denervation, independent of the mechanical environment, has been replicated in subsequent animal (23, 24), clinical (25$27)$, and computational analysis $(28,29)$ studies. Therefore, our previous data indicate that defects in muscle growth are the initiating cause of contractures. Understanding how denervation impairs longitudinal muscle growth could open the door to novel biological therapies to preserve muscle growth, ultimately replacing the destructive palliative therapies currently used to treat contractures.

Interestingly, the overstretched sarcomeres following NBPI are identical to those seen in human muscles responsible for contractures in CP (30). Although animal models of CP-induced contractures have never been successfully created (31-33), the use of an NBPI model to understand the mechanism by which longitudinal muscle growth is mediated and impaired could offer novel targets and strategies to prevent neuromuscular contractures in both NBPI and CP. Importantly, we have found in the NBPI model that contractures do not occur following muscle denervation outside the neonatal period (18), consistent with the clinical observations that BPI in later childhood does not cause contractures (34), and suggesting a unique biologic susceptibility of neonatal longitudinal muscle growth to denervation. However, in contrast with the vast knowledge of the mechanisms that regulate muscle width, the processes in muscle that govern muscle length during the neonatal period are unexplored.

In general, muscle grows by 2 basic processes: (a) fusion of muscle stem cells (MuSCs) (35) to growing multinucleated myofibers (myonuclear accretion), and (b) an anabolic balance between protein synthesis and protein degradation within the myofibers. The contributions of these mechanisms to longitudinal muscle growth have not been experimentally dissected. A central role has been assumed for myonuclear accretion in both neonatal muscle growth and contracture development, since myonuclear accretion is unique to neonatal muscle growth $(36,37)$, and since others have found MuSC depletion following longterm denervation (38) or in longstanding contractures from CP (39-42). However, these latter findings have been based on analyses of muscles obtained after contractures have formed, so causation cannot be determined. Thus, major questions remain about the relative contributions of myonuclear accretion, protein synthesis, and protein degradation to muscle growth and contractures during the time frame of neonatal neuromuscular contracture development.

In this work we utilize an established mouse model of NBPI that reliably causes shoulder and elbow contractures that begin 2 weeks following denervation and plateau at 4 weeks (21), and which can be measured using a previously validated technique with excellent reliability (intraclass correlation coefficient $=0.97,95 \%$ CI from 0.92 to 0.99 ) and a standard error of measurement of $3^{\circ}(16)$. While contractures at both the shoulder and elbow are evaluated as phenotypic outcomes, the current investigations regarding contracture etiology are focused on the elbow flexion contracture, since the elbow has simpler motion, musculature, and innervation than the shoulder, allowing clearer interpretation 
of results delineating the relationships between muscle pathology and contracture phenotype. With this model, herein we have found that neonatal denervation does not prevent myonuclear accretion, and that inhibiting myonuclear accretion does not impair longitudinal muscle growth. These findings rule out a role for myonuclear accretion in longitudinal muscle growth and contracture development. Furthermore, we found that denervation causes elevation in both protein synthesis and protein degradation, only the latter of which could explain reduced muscle growth. Importantly, we discovered that inhibition of proteasome-mediated protein degradation restores muscle length and prevents contractures following NBPI, identifying a cellular mechanistic underpinning of contracture pathogenesis and uncovering what we believe is a novel strategy to prevent neonatal neuromuscular contractures.

\section{Results}

Dysregulation of MuSCs during contracture development. Because a unique property of neonatal muscle is the high rate of fusion between muscle progenitors and myofibers that ultimately increases myonuclear numbers (36), we assessed whether MuSC dysregulation could contribute to contracture pathogenesis. It has been previously shown that MuSC numbers are reduced in muscle after neonatal denervation (38) and in CP $(39,42)$, although these analyses were performed after the time period in which contractures are established, leaving it unclear whether dysregulation of MuSCs are a cause or consequence of the pathology. We thus investigated quiescent and activated MuSC populations before and during contracture development in our established murine model of NBPI, where unilateral surgical excision of the brachial plexus (nerve roots C5-T1) in postnatal day 5 (P5) mice results in forelimb muscle denervation and reliably causes contractures in the shoulder and elbow consistent with the human phenotype within 4 weeks after NBPI $(16,18)$. We first immunostained for Pax7, a marker of MuSCs, in contralateral (normally innervated) and NBPI (denervated) biceps muscles 2 weeks after denervation and observed elevated levels of Pax $7^{+}$ cells in NBPI muscle (Figure 1A). We further assessed the MuSC populations by immunostaining biceps sections for Pax7 and MyoD, a marker for activation of the myogenic program (Supplemental Figure 1A; supplemental material available online with this article; https://doi.org/10.1172/jci.insight.128454DS1), and by quantifying the percentage of MuSCs that were $\mathrm{Pax} 7^{+} \mathrm{MyoD}^{-}$(quiescent), $\mathrm{Pax} 7^{+} \mathrm{MyoD}^{+}$(activated), and Pax7-MyoD ${ }^{+}$(differentiated). We found the same levels of activated and differentiated MuSCs in contralateral and NBPI muscle, which were expectedly low in both groups given that the analysis was performed toward the end of the developmental window, but we found an increase in quiescent cells in NBPI muscle (Figure 1B), suggesting MuSC dysregulation. One possibility to explain the abundance of quiescent MuSCs is a block to activation/proliferation, which could also conceptually explain impaired muscle growth. We therefore performed unilateral NBPI on P5 wild-type (WT) mice and treated them with 5-bromo-2'-deoxyuridine (BrdU) for 2 weeks (Figure 1C). The number of Pax $7^{+}$cells incorporating BrdU at 2 weeks after NBPI was increased compared with the contralateral muscle (Figure 1D), ruling out a block to proliferation among MuSCs. These data together indicate that while MuSCs exhibit aberrant properties in neonatally denervated muscle, they are present and capable of proliferation and differentiation.

Still, another mechanism by which MuSC dysregulation could impact muscle length is altered myonuclear accretion, leading to myonuclear numbers that are insufficient for building sarcomeres and establishing muscle length. To assess myonuclear accretion in NBPI, we used the same BrdU-labeling protocol mentioned above (Figure 1C), but assessed $\mathrm{BrdU}^{+}$nuclei within a dystrophin ${ }^{+}$myofiber as an indicator of fusion of new nuclei, because myonuclei already within the myofiber are not proliferative and are unable to incorporate BrdU. Denervated muscle 2 weeks after NBPI exhibited increased percentages of myofibers containing $\mathrm{BrdU}^{+}$myonuclei compared with the contralateral side (Figure 1E). To complement this approach, we also genetically labeled MuSCs and tracked their incorporation into the myofiber by crossing the MuSC-specific tamoxifen-inducible Pax $7 C^{\mathrm{CrER}}$ mouse with a Rosa $26^{\mathrm{LacZ}}$ reporter. Pax ${ }^{\text {CreER }}{ }^{\mathrm{R} o s a 26^{\mathrm{LacZ}}}$ mice were subjected to NBPI at P5, treated with tamoxifen at P7, and analyzed for $\mathrm{LacZ}^{+}$myofibers at P19 (Supplemental Figure 1B). X-gal staining of sections revealed $\mathrm{LacZ}^{+}$myofibers in both contralateral and NBPI muscle, and quantification revealed an increased percentage of $\mathrm{LacZ}^{+}$fibers 2 weeks after NBPI (Supplemental Figure 1C). These data suggest that myonuclear accretion is increased rather than decreased in denervated muscle.

Reduced myonuclear accretion does not impair longitudinal muscle growth or induce contractures. Because our findings suggesting normal or increased MuSC numbers and activity during the time frame of contracture pathogenesis are in contrast with others' findings indicating fewer MuSCs $(39,43)$ with less in vitro myogenic capacity (44) after contractures have formed, we next needed to experimentally manipulate 
MuSC-mediated myonuclear accretion to definitively outline the role of myonuclear accretion in longitudinal muscle growth and contractures. Therefore, we blocked myonuclear accretion through genetic deletion of myomaker (Mymk), a muscle-specific protein required for muscle progenitor fusion (45), specifically in MuSCs during the early postnatal period. We treated Mymk $k^{\operatorname{loxP} / \operatorname{loxP}}$ (control) and Mymk $k^{\operatorname{loxP} / \operatorname{loxP}}$ Pax CreER $\left(M y m k^{\text {scKO}}\right)$ mice $(46,47)$ with tamoxifen at P0 and found significant downregulation of Mymk expression in muscle at P5 (Figure 2A). Moreover, a 75\% reduction in nuclear number in hindlimb myofibers at P28 was observed (Figure 2, B and C), establishing that experimental manipulation of myonuclear accretion can be achieved during the time frame of contracture formation following NBPI at P5. Of note, the reduced myonuclear number in $M y m k^{\text {scKo }}$ myofibers was characterized by an increased myonuclear domain per unit length, measured in sarcomeres per nucleus over 1000- $\mu \mathrm{m}$ segments of the myofiber (Figure 2D). This increase in myonuclear domain in length was not simply due to narrower diameters of the myofibers, given a concomitant increase in myonuclear domain in volume (Figure 2D). These data indicate that sarcomere addition can occur in series without the full complement of myonuclear number.

Having established our ability to limit myonuclear accretion during the relevant developmental window, we utilized the $M y m k^{\text {scKO }}$ model to directly evaluate if reduced myonuclear numbers would cause contractures at baseline or exacerbate the NBPI contracture phenotype. Control and Mymk $k^{\text {scKo }}$ mice were treated with tamoxifen at P0, followed by unilateral NBPI at P5, and mice were harvested at P33 (4 weeks after NBPI) (Figure 2E). Single myofibers from the biceps were analyzed for numbers of myonuclei, which revealed that deletion of Mymk caused the expected reduction in nuclei per myofiber in both contralateral and NBPI muscle (Figure 2, F and G). We did not observe a significant reduction in myonuclei in control NBPI biceps compared to control contralateral biceps, although a small reduction may be present. Regardless, this finding suggests that denervation alone is insufficient to substantially reduce myonuclear number, which is consistent with our finding of maintained myonuclear accretion in denervated muscle.

We then determined if reduction in myonuclear numbers impacts whole muscle length and development of contractures. Measurement of whole muscle functional length is complicated by the fact that muscle is composed of a series of elastic elements: the sarcomeres. True functional whole muscle length is defined by the number of sarcomeres in series, which cannot be directly measured in a whole muscle. Moreover, because apparent gross whole muscle length varies relative to the degree of muscle stretch, simply measuring the gross muscle length is insufficient to determine functional muscle length. Similarly, measurement of gross muscle length is confounded by animal size as well as altered mechanical properties of slack muscle from fibrosis, confounding variables that can vary among our different experimental conditions and thus cannot be appropriately controlled. Finally, individual myofiber length does not necessarily describe whole muscle functional length, given varied pennation angles and myofiber overlap within a whole muscle. However, a muscle's relative total sarcomere number in series (or relative functional length) can be estimated by measuring the muscle's average sarcomere length at controlled stretch: the fewer sarcomeres a muscle has in series, the more each sarcomere has to stretch to accommodate a given position of the joint(s) that the muscle crosses. For instance, the brachialis muscle, an elbow flexor, can be held at a controlled stretch by controlling the elbow joint position. If a given brachialis muscle has elongated sarcomeres when compared with another brachialis muscle positioned at an identical elbow position, the muscle with the elongated sarcomeres is interpreted to have fewer sarcomeres in series. Several confounding variables and assumptions must be addressed for this relationship to hold true. First, since sarcomere number can change with developmental growth, only limbs/ animals at the same developmental age can be compared. Second, since skeletal architecture can alter the stretch applied to a muscle, bony anatomy must be unaltered among the conditions being compared. Finally, the assumption is made that the overall muscle architecture and shape (pennation angle, vector of pull) does not vary among the conditions being compared; thus, only identical muscles can be compared directly. Nonetheless, these confounders can be overcome by comparing identically aged, symmetrically positioned samples of the same muscle, with radiographic assessment for bony deformity, as was done in our study. With this analysis, we found that sarcomere length was unchanged in contralateral (normally innervated) muscles of control and $M y m k^{\mathrm{scKO}}$ mice, and while NBPI resulted in increased sarcomere length (reduced functional muscle length) in both groups of mice, loss of Mymk and reduction in myonuclear number did not exacerbate the pathology (Figure 2H). Therefore, denervation affected brachialis functional muscle length, but Mymk deletion did not. Similarly, NBPI significantly reduced passive elbow extension in both groups, but Mymk deletion did not worsen the reduction in range of motion caused by NBPI or reduce the range of motion on the contralateral side (Figure 2I). In contrast with the apparent reduction in myofiber diameter in hindlimb 
A Contralateral

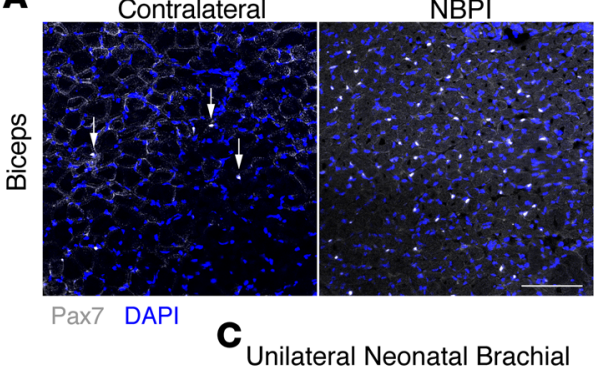

Plexus Inury (NBPI)

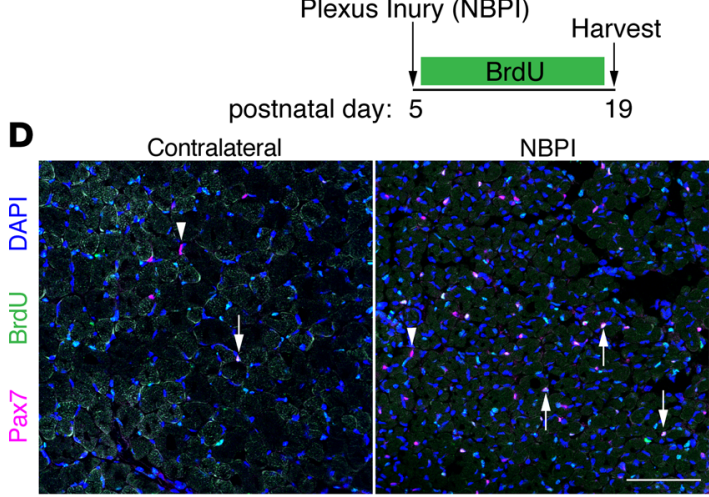

$\mathbf{E}$

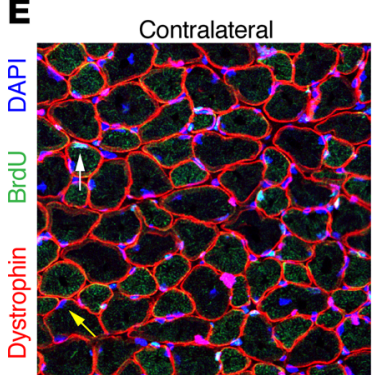

NBPI

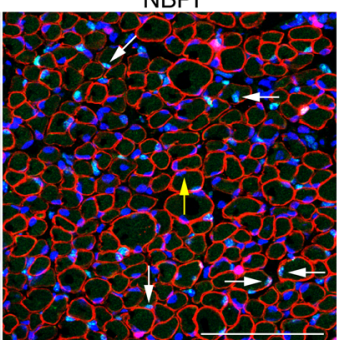

B

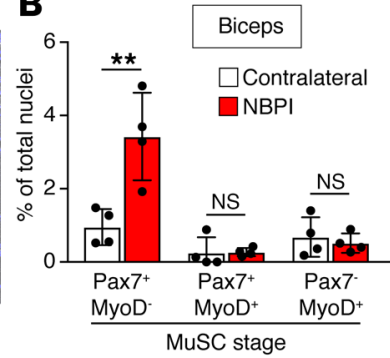

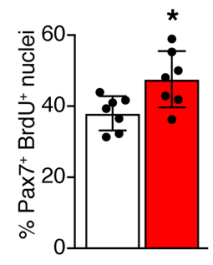

$\square$ Contralateral $\square$ NBPI

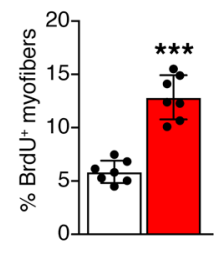

Figure 1. Muscle stem cell dysregulation during development of neonatal contractures. (A) Immunohistochemistry for Pax7 in biceps from contralateral and 2 weeks after neonatal brachial plexus injury (NBPI). Arrows indicate Pax $7^{+}$ cells. (B) Quantification of biceps sections immunostained with Pax7 and MyoD antibodies to assess stage of muscle stem cell (MuSC) quiescence and activation. The number of Pax7 $7^{+} \mathrm{MyoD}^{-}$(quiescent), Pax7 $7^{+} \mathrm{MyoD}^{+}$(activated), and Pax7-MyoD+ (differentiated) cells were normalized to total nuclei ( $n=4$ each for contralateral and NPBI). (C) Experimental scheme for BrdU treatment during the initial 2 weeks after NBPI. (D) Representative images (left) of immunostaining with Pax7 and BrdU antibodies in contralateral and NBPI muscle. Arrows show Pax $7^{+} \mathrm{BrdU}^{+}$cells and arrowheads

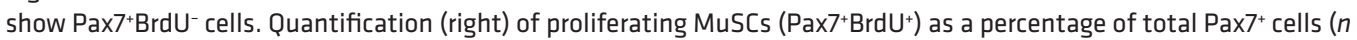
= 7 each for contralateral and NBPI). (E) Representative images (left) showing BrdU+ myonuclei, defined as being BrdU ${ }^{+}$ and entirely within a dystrophin ${ }^{+}$myofiber, as an indicator of myonuclear accretion. White arrows indicate a BrdU ${ }^{+}$ myonucleus, whereas yellow arrows show a BrdU- myonucleus. Quantification (right) of the percentage of myofibers containing a BrdU+ nucleus ( $n=7$ each for contralateral and NBPI). Data are presented as mean \pm SD. Because all comparisons were done to the contralateral, unoperated forelimbs, statistical analyses were performed with paired, 2-tailed Student's $t$ tests except for B where Wilcoxon's signed-rank test was used for Pax7 ${ }^{+}$MyoD $D^{+}$biceps due to non-normal distributions. ${ }^{*} P<0.05,{ }^{* *} P<0.01,{ }^{* *} P<0.001$. Scale bars: $100 \mu \mathrm{m}$. NS, not significant.

muscles (Figure 2, B-D), reduced myonuclear number from Mymk deletion did not significantly worsen the biceps myofiber width reduction from denervation or reduce biceps myofiber width on the contralateral side (Figure $2 \mathrm{~J}$ ). Therefore, the role of myonuclear accretion in regulation of myofiber width may vary between muscles. However, the data here show that reducing myonuclear number does not elicit defects in whole muscle length or cause contractures, indicating that myonuclear number does not control longitudinal muscle growth or NBPI-induced contractures.

Neonatally denervated muscle is characterized by altered protein balance. Having eliminated myonuclear accretion, or myonuclear number, as a relevant mechanism in longitudinal muscle growth and contractures, we hypothesized that impaired muscle growth could be explained by reduced protein synthesis 
A

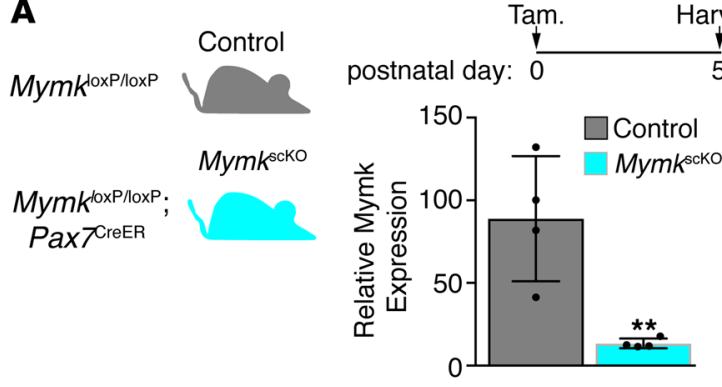

D

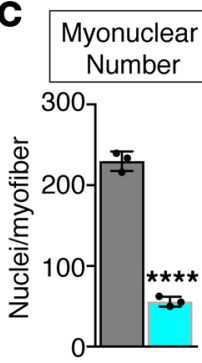

E Mymk $k^{\text {loxP } / \text { oxP }}$

Mymk koxPloxP; Pax 7 CreER

Unilateral Neonatal Brachial Plexus Inury (NBPI)

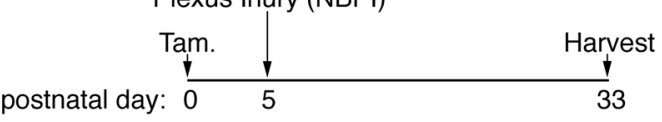

Control

$M_{y m k}^{\text {scko }}$

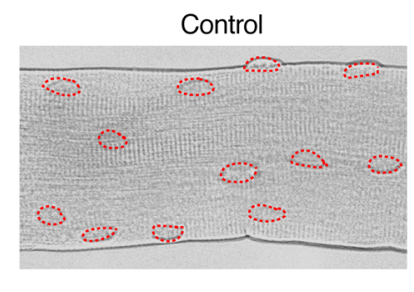

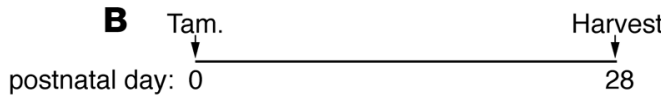

28

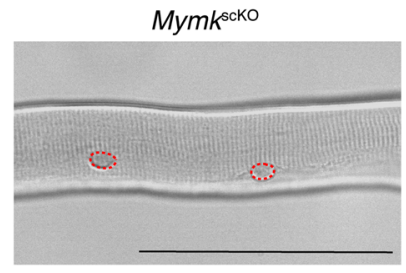

Extensor Digitorum Longus (EDL)

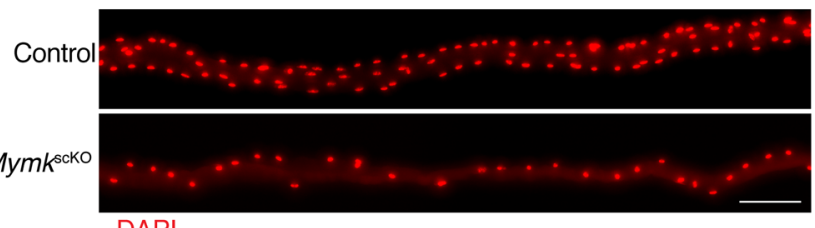

DAPI
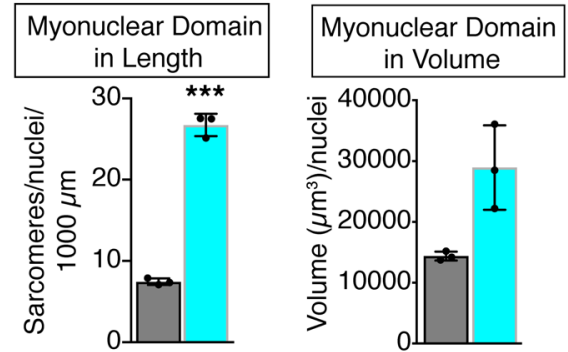

$\mathbf{F}$

Biceps

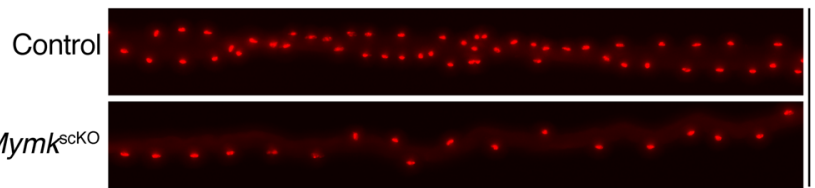

Contralateral

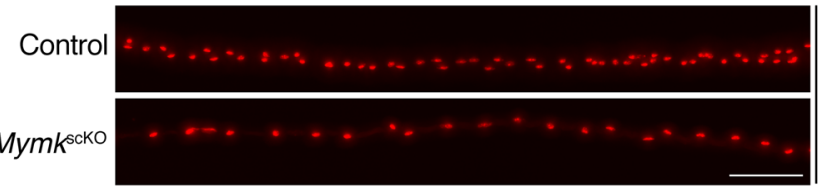

NBPI

DAPI
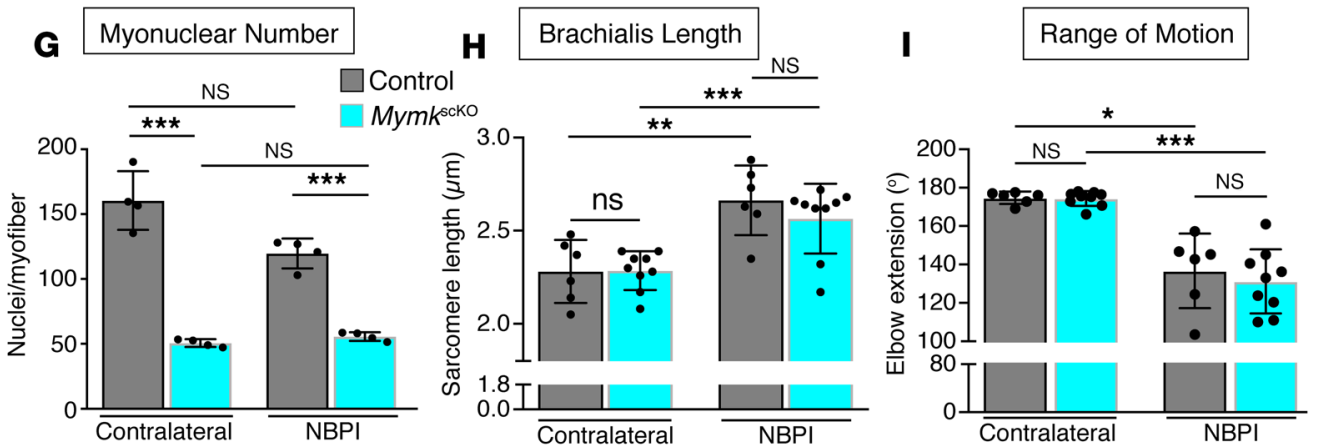

J Biceps Myofiber Width

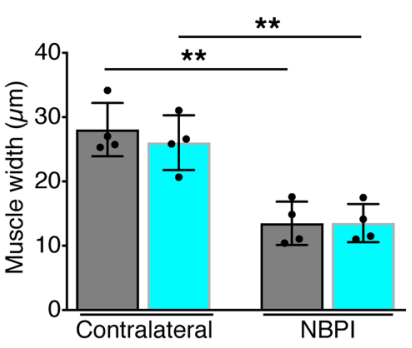

Figure 2. Reduced myonuclear numbers do not control muscle length or contracture pathology. (A) Mymk (myomaker) was deleted in MuSCs to prevent myonuclear accretion. Expression of Mymk in muscle from Mymk $k^{\text {loxP/loxP }}$ (control) and Mymk ${ }^{\text {loxP/loxP Pax } 7 \text { reeR }}$ (Mymk $k^{\text {scko }}$ ) at postnatal day 5 (P5) after treatment with tamoxifen (Tam.) at PO ( $n=4$ each for control and Mym $k^{\text {scko }}$ ). (B) Representative single myofibers from the extensor digitorum longus (EDL), stained with DAPI, of control and $M y m k^{\mathrm{sck} 0}$ at P28, following tamoxifen at PO. (C) Quantification of nuclei per myofiber from the samples in B ( $n=3$ each for control and Mymk $k^{\text {scko }}$ ). (D) DIC images (left) from control and Mym $k^{\mathrm{sck} 0} \mathrm{EDL}$ showing similar sarcomere lengths. Nuclei are outlined in red. Quantification (right) of the myonuclear domain in length, expressed as the number of sarcomeres per nucleus in a 1000- $\mu \mathrm{m}$ segment of the myofiber; and quantification of myonuclear domain in volume ( $n=$ 3 each for control and $M y m k^{\text {scko }}$. (E) Schematic showing experimental design to delete Mymk just before NBPI and assess myonuclear numbers and contracture pathology at P33. (F) Single myofiber images from contralateral and NBPI biceps of control and Mymk ${ }^{\text {scko }}$ mice. DAPI shows myonuclei. (G) Quantification of nuclei per myofiber in the various groups of mice $\left(n=4\right.$ each for control and $\left.M y m k^{\mathrm{sck} 0}\right)$. (H) Brachialis sarcomere length, where increased sarcomere length indicates reduced functional muscle length (sarcomeres in series). Reduction in myonuclear numbers by $75 \%$ in $M y m k^{\text {scko }}$ muscle does not impact muscle length (control, $n$ $=6$ and $M y m k^{\text {scko }}, n=9$ ). (I) Assessment of elbow extension in the various groups of mice, where $170^{\circ}-180^{\circ}$ represents full range of motion. NBPI causes reduced range of motion, but reduction in myonuclear numbers in $M y m k^{\text {scko }}$ does not reduce range of motion in contralateral limbs or exacerbate the reduction caused by NBPI (control, $n=6$ and $M y m k^{s c k 0}, n=9$ ). (J) Assessment of biceps average single myofiber widths from F. NBPI reduces myofiber width, but reduction in myonuclear numbers in Mymk $k^{\mathrm{sck0}}$ does not reduce myofiber width in contralateral biceps or exacerbate the reduction caused by NBPI (control, $n=4$ and $\left.M y m k^{\mathrm{sck} 0}, n=4\right)$. Data are presented as mean \pm SD. Statistical analysis was performed with unpaired 2-tailed Student's $t$ test in A, C, and D; and with unpaired, 2-tailed Student's $t$ test between groups and paired, 2-tailed Student's $t$ test between limbs of mice in each group in G-J; except comparisons including NBPI brachialis sarcomere length in Mymk $^{\mathrm{sck} 0}$ mice in $\mathbf{H}$, where nonparametric tests (Mann-Whitney $U$ test between groups and Wilcoxon's signed-rank test between sides) were used due to non-normal distribution of these data. Bonferroni's corrections for multiple comparisons were performed in G-J, and adjusted $P$ values are reported for those data. ${ }^{*} P<0.05,{ }^{* *} P<0.01,{ }^{* * *} P<0.001,{ }^{* * *} P<0.0001$. Scale bars: $100 \mu \mathrm{m}$. NS, not significant. 
A

Gene Ontology Biological Processes (336 up-regulated genes 2w NBPI)

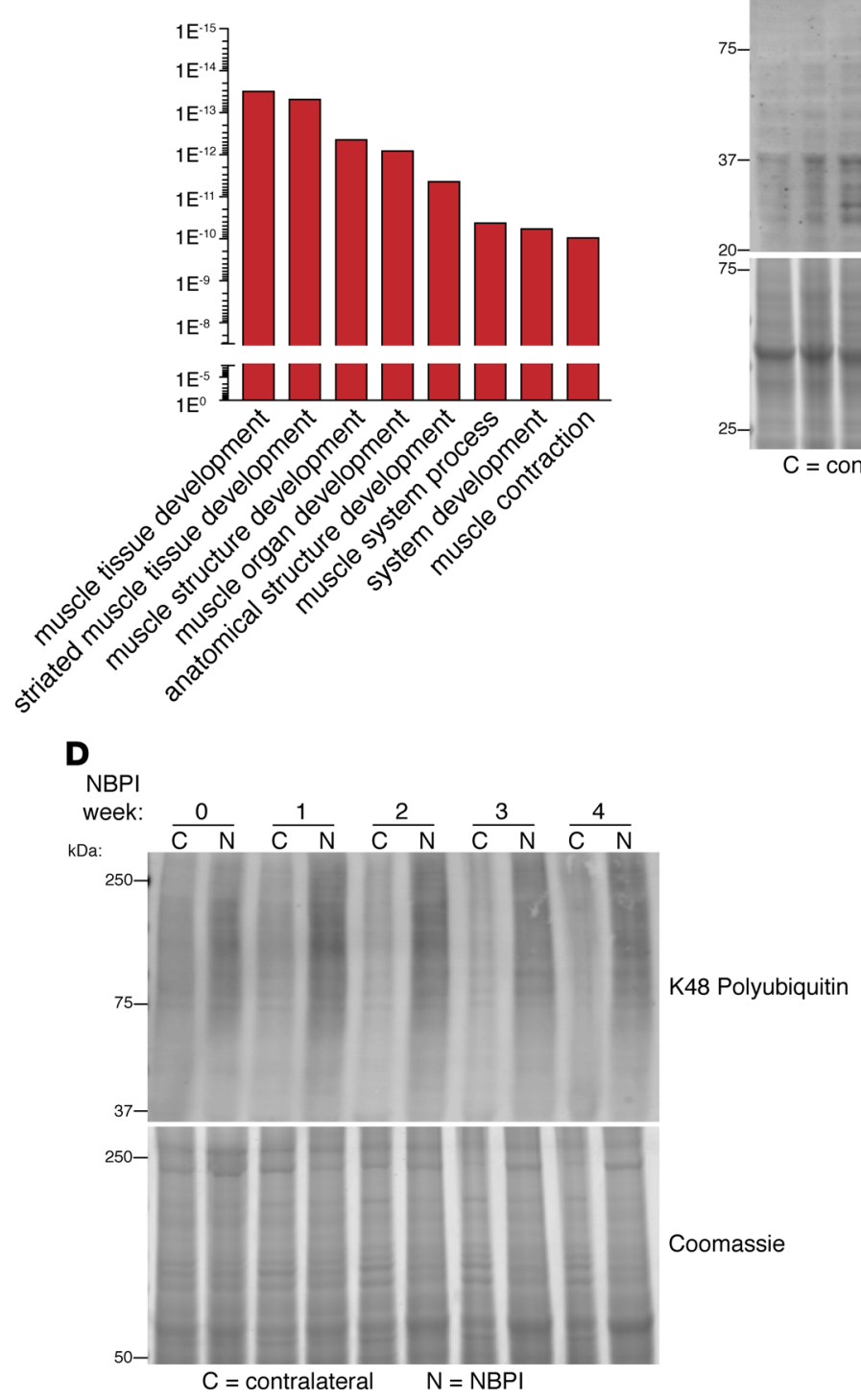

B
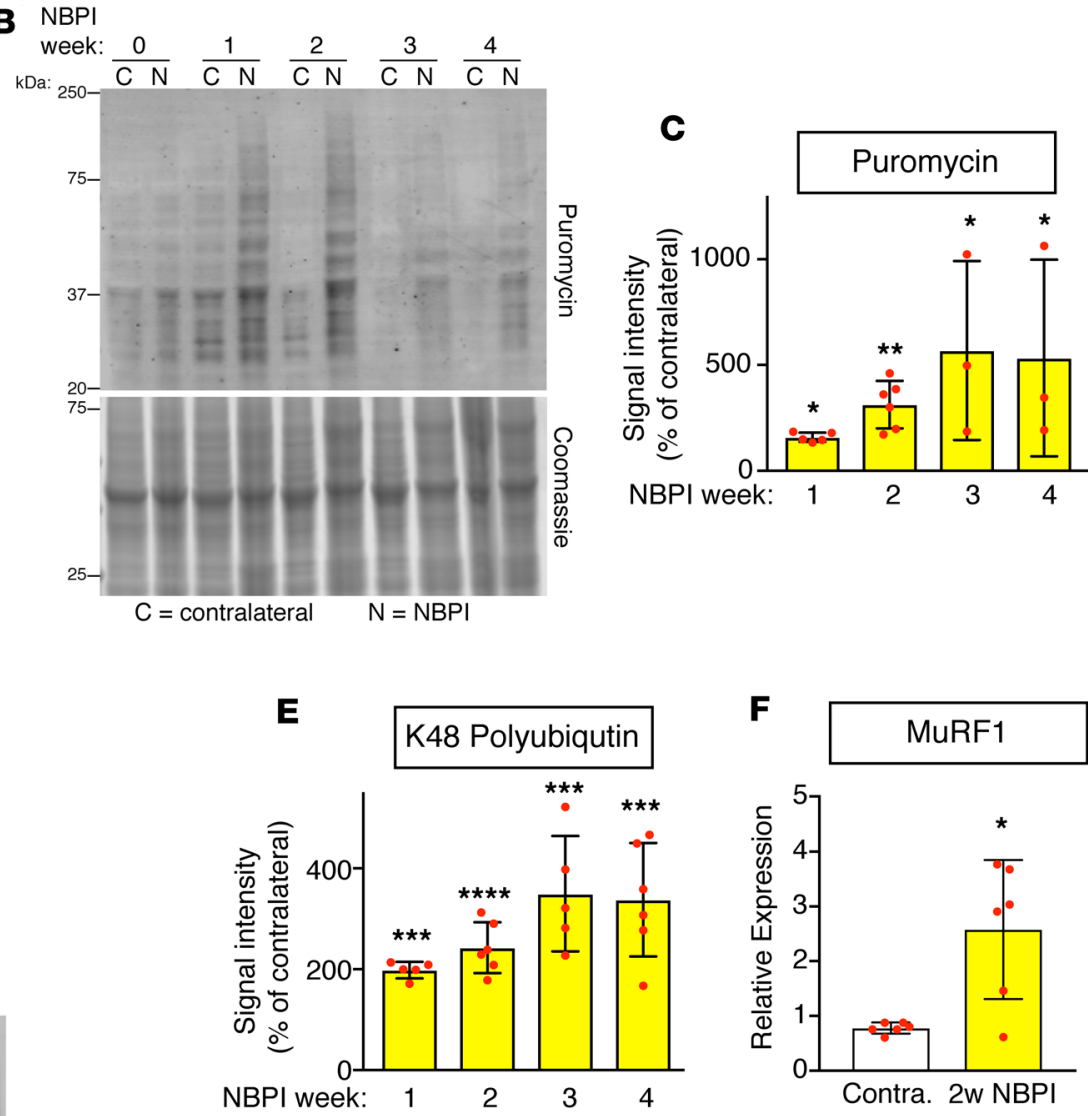

Figure 3. Elevated protein degradation in NBPI muscle. (A) Gene ontology analysis of the 336 genes upregulated in muscle 2 weeks (2w) after NBPI. (B) Analysis of protein synthesis in muscle after mice were treated with puromycin, which is incorporated into nascent polypeptides. Shown is a representative puromycin Western blot of muscle samples from various NBPI time points. Coomassie was used as a loading control. (C) Quantification of the puromycin signal in NBPI muscle in B expressed as a percentage of the contralateral (week $1 n=5$, week $2 n=6$, week $3 n=3$, week $4 n=3$ ). (D) Representative Western blot for K48-polyubiquitin where Coomassie is shown as a loading control. (E) K48-polyubiquitin signal intensity at multiple weeks after NBPI, expressed as a percentage of the contralateral (week $1 n=5$, week $2 n=6$, week $3 n=5$, week $4 n=6$ ). (F) MuRF1 transcript levels 2 weeks after NBPI (contralateral $n=6,2 \mathrm{w} \mathrm{NBPI} n=6$ ). (C) Fluorescence-based assay for 205 proteasome activity, normalized to amount of protein (contralateral $n=6$, 2w NBPI $n=6$ ). Data are presented as mean \pm SD. Statistical analysis was performed with paired, 2-tailed Student's $t$ test comparing NBPI muscle to contralateral. ${ }^{*} P<0.05,{ }^{* *} P<0.01,{ }^{* *} P<0.001,{ }^{* * *} P<0.0001$. See complete unedited blots in the supplemental material.

or increased protein degradation. We performed RNA sequencing on contralateral and NBPI muscle 3 weeks after surgery and found 336 upregulated and 21 downregulated genes. Gene ontology analysis revealed that denervation causes upregulation of genes predominantly related to muscle development and structure (Figure $3 \mathrm{~A}$ ), suggesting that denervated muscle is transcriptionally competent. We then tested if denervated muscle is able to synthesize protein at the translational level, as assessed through puromycin incorporation into nascent polypeptides, at multiple time points after NBPI. We observed normal protein synthesis in NBPI muscle just after denervation (week 0) but an increase compared with contralateral muscle at all later time points (Figure 3, B and C; see complete unedited blots in the supplemental material). 
Thus, protein synthesis is elevated following NBPI, which conceptually cannot explain the mechanism of contracture pathology since increased protein synthesis should allow more muscle growth.

We next employed multiple approaches to evaluate protein degradation, a process known to be activated in adult denervated muscle. Indeed, the ubiquitin/proteasome pathway accounts for $90 \%$ of the protein breakdown in adult denervation-induced muscle atrophy (48). We discovered elevated K48-polyubiquitinated proteins in denervated muscle at all time points after NBPI (Figure 3, D and E; see complete unedited blots in the supplemental material). Additionally, in neonatally denervated muscle we observed increased expression of muscle ring-finger protein-1 (MuRF1) (Figure 3F), a muscle-specific E3 ubiquitin ligase that is a central factor eliciting the cascade of protein degradation in muscle (49). Finally, using a commercially available assay for the chymotrypsin-like catalytic activity of the $20 \mathrm{~S}$ proteasome (50), we found increased proteasome activity in denervated muscle 2 weeks after NBPI (Figure 3G). Overall, multiple points in the protein degradation pathway are increased following NBPI, which could explain the impaired growth of neonatally denervated muscle.

Pharmacological inhibition of the proteasome prevents contractures. We therefore tested if pharmacologic inhibition of proteasome-mediated protein degradation after NBPI could preserve muscle length and prevent contractures. Following NBPI at P5, the 20S proteasome inhibitor, bortezomib (51), was administered at a dose of $0.4 \mathrm{mg} / \mathrm{kg}$ body weight every other day from P5 to P33 (Figure 4A). Bortezomib was coadministered with [Gly14]-Humanin G ([Gly14]-HN) to mitigate known toxic effects of bortezomib (52). Saline and [Gly14]-HN were administered individually in separate animals as controls. Blinded assessment of shoulder and elbow range of motion in mice 4 weeks after NBPI indicated that bortezomib rescued the elbow and shoulder contracture phenotypes (Figure 4B), significantly reducing shoulder and elbow contracture severity (difference between NBPI and contralateral forelimb passive external rotation and elbow extension, respectively) (Figure 4C). [Gly14]-HN had no effect alone. However, bortezomib treatment caused mortality, mostly in the first week of treatment (Supplemental Figure 2A). To overcome this toxicity we optimized the dose and timing of bortezomib. Specifically, we treated WT mice with bortezomib using the following regimens: $0.2 \mathrm{mg} / \mathrm{kg}$ from P5 to P33, $0.3 \mathrm{mg} / \mathrm{kg}$ from P5 to P33, $0.4 \mathrm{mg} / \mathrm{kg}$ from P8 to P33, and $0.4 \mathrm{mg} / \mathrm{kg}$ from P12 to P33 (Supplemental Figure $2 \mathrm{~B}$ ). Lowering the dose to $0.2 \mathrm{mg} / \mathrm{kg}$ or delaying treatment until P12 eliminated mortality (Supplemental Figure 2C), but while these strategies resulted in less severe contractures compared with saline they were not as efficacious as $0.4 \mathrm{mg} / \mathrm{kg}$ bortezomib administered beginning on P5 (Supplemental Figure 2D). Conversely, lowering the dose to $0.3 \mathrm{mg} / \mathrm{kg}$ or initiating treatment on P8 maintained efficacy and partially improved mortality compared with $0.4 \mathrm{mg} / \mathrm{kg}$ bortezomib administered on P5 (Supplemental Figure 2, C and D).

Using the above bortezomib data, we optimized a dosing strategy to maximize efficacy and limit mortality. We treated WT mice with $0.3 \mathrm{mg} / \mathrm{kg}$ bortezomib from P8 to P33 (Figure 4D). With this treatment strategy, we observed minimal early death (Supplemental Figure 2E), normal body weight gain (Supplemental Figure 2F), and no effect on humerus bone length (Supplemental Figure 2G), but we observed optimal efficacy in prevention of contractures (Figure 4, E and F). This therapeutic effect of bortezomib was accompanied by a rescue of brachialis length, as evidenced by a $70 \%$ reduction in the sarcomere elongation caused by NBPI (Figure 4G), further indicating that neonatal contractures are caused by impaired longitudinal muscle growth. This rescue of sarcomere length occurred without rescue in whole muscle cross-sectional area (Figure $4 \mathrm{H}$ ) or volume (Figure 4I), consistent with prior reports of modest improvements in muscle mass following bortezomib treatment of adult denervated muscle (53), and consistent with potentially differential effects of protein degradation in regulation of muscle length versus cross-sectional area. Importantly, this optimized dose of bortezomib blunted the denervation-induced increase in proteasome activity at 2 weeks after NBPI, during the time frame of contracture development, consistent with the drug's mechanism of action and supporting a role for protein degradation in contracture pathogenesis (Figure $4 \mathrm{~J}$ ). In total, the findings presented here show that bortezomib preserves length of denervated muscle and prevents contractures in a dose-dependent manner following NBPI, representing what we believe is the first ever strategy to prevent neuromuscular contractures by correcting the underlying pathology.

\section{Discussion}

For decades, neuromuscular contractures have been considered a mechanical problem absent any biological explanation, and only palliative mechanical solutions for them have been available. In this work, we demonstrate that the fundamental mechanism leading to contracture development is improper longitudinal muscle growth due to increased proteasome activity. Surprisingly, MuSCs and myonuclear accretion do not control 
A

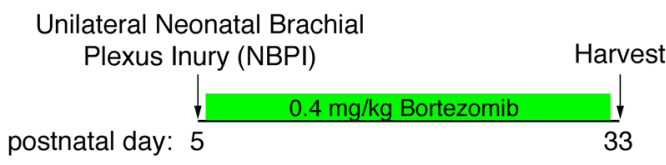

B

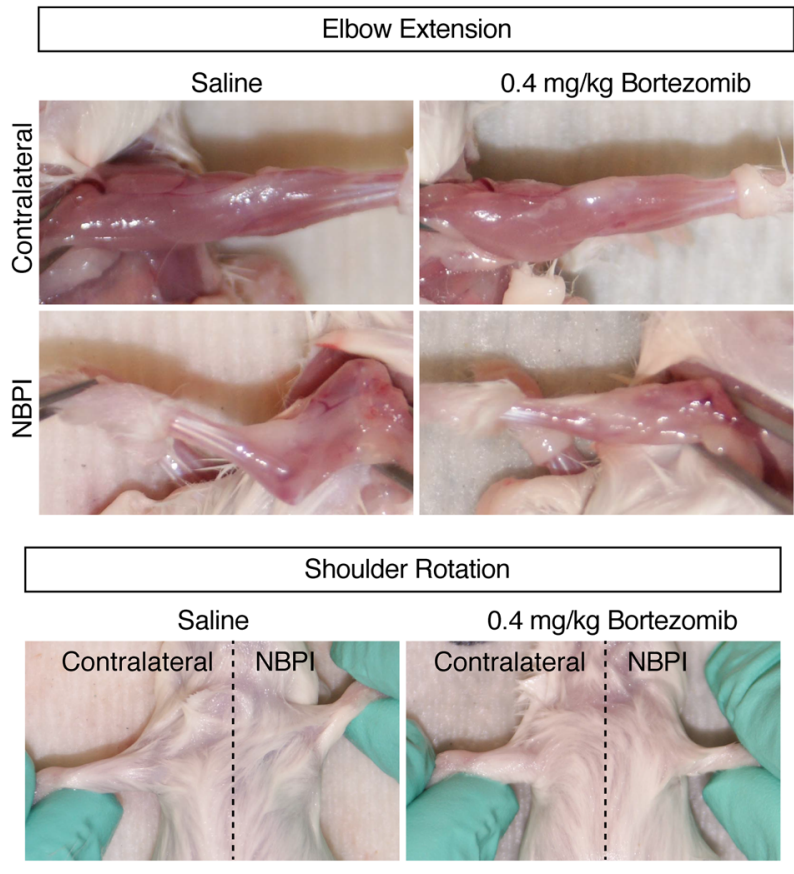

E
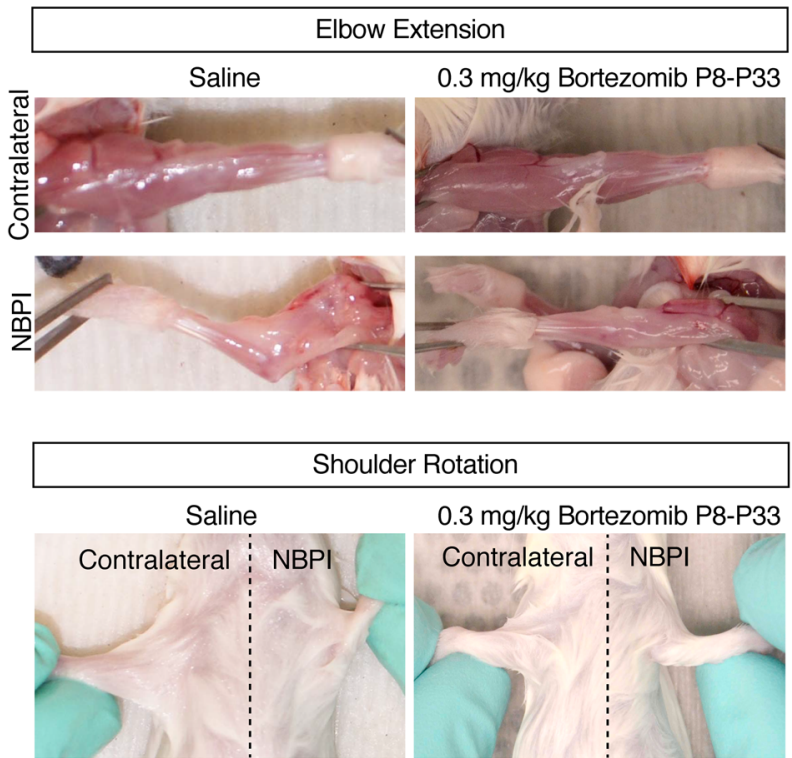
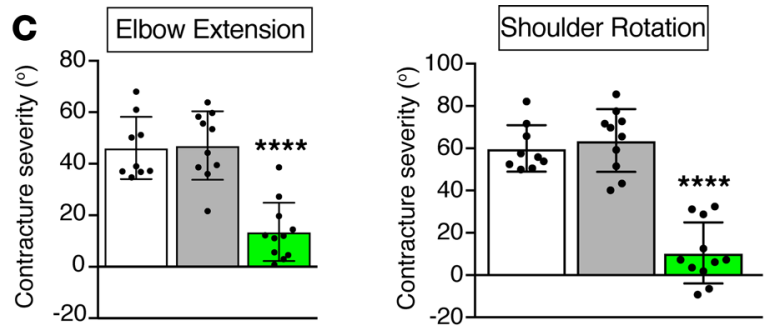

D
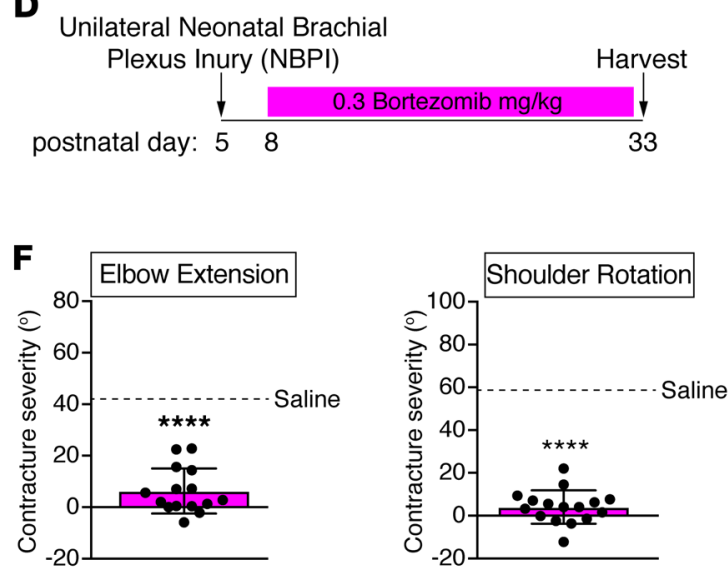

$\square$ Saline

$\square$ [Gly14]-HN

$0.3 \mathrm{mg} / \mathrm{kg}$ Bortezomib P8-P33
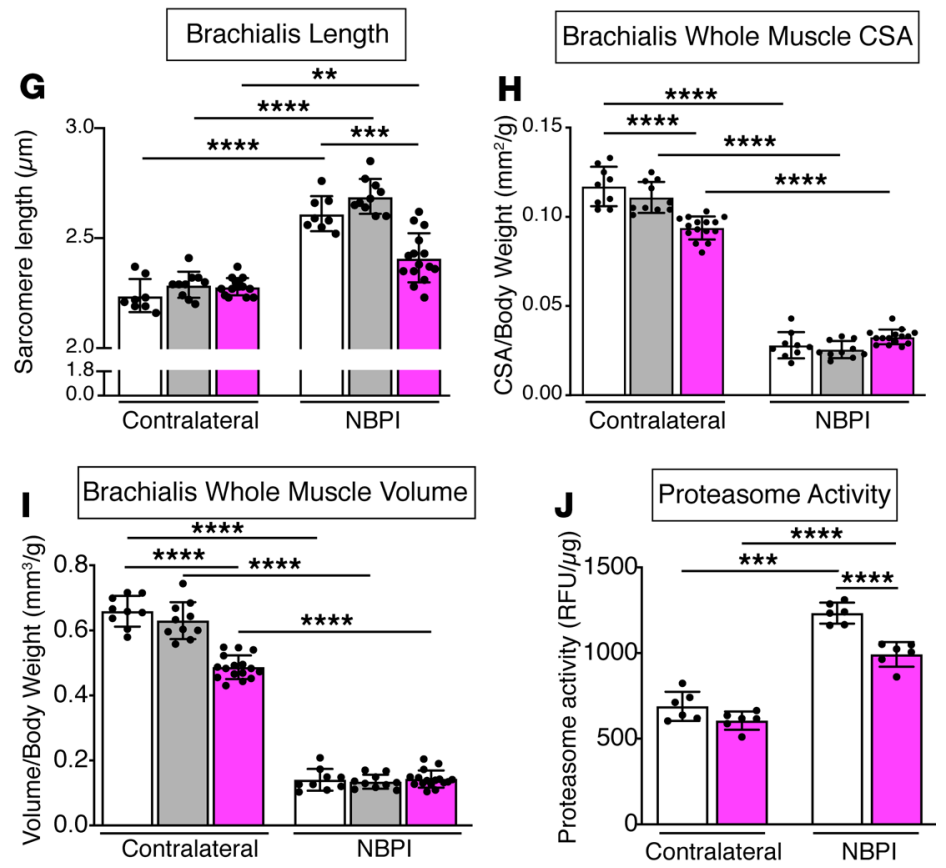

Figure 4. Pharmacologic inhibition of the proteasome preserves longitudinal muscle growth and prevents contractures. (A) Experimental scheme for NBPI and bortezomib treatment. (B) Images of forelimbs showing contractures in elbow (top) and shoulder (bottom) after NBPI, which are corrected with bortezomib. (C) Quantification of contracture severity, calculated as the difference in extension (elbow) or rotation (shoulder) between NBPI and contralateral. Saline and [Gly14]-Humanin G ([Gly14]-HN) were used as controls (saline $n=9$, [Gly14]-HN $n=10,0.4$ mg/kg bortezomib $n=11$ ). (D) Schematic showing the optimized bortezomib treatment strategy. (E) Forelimb images showing the lack of contractures after NBPI in mice treated with 0.3 mg/ kg bortezomib beginning on P8. (F) Contracture severity in the elbow and shoulder from the mice shown in $\mathbf{E}$. The dotted line represents the severity in saline controls (from C) (elbow $n=15$, shoulder $n=16$ ). (G) Sarcomere length in the brachialis shows that 0.3 mg/kg bortezomib preserves muscle length (saline $n=9$, [Gly14]-HN $n=10,0.3 \mathrm{mg} / \mathrm{kg}$ bortezomib $n=15$ ). (H) Whole muscle cross-sectional area (CSA) of brachialis muscles shows that bortezomib does not rescue muscle CSA (saline $n=9$, [Gly14]-HN $n=10,0.3 \mathrm{mg} / \mathrm{kg}$ bortezomib $n=15$ ). (I) Whole muscle volume of brachialis muscles shows that bortezomib does not rescue muscle volume (saline $n=9$, [Gly14]-HN $n=10,0.3 \mathrm{mg} / \mathrm{kg}$ bortezomib $n=16$ ). (J) Proteasome activity in denervated 
biceps muscles harvested 2 weeks after treatment with saline $(n=6)$ and $0.3 \mathrm{mg} / \mathrm{kg}$ bortezomib $(n=6)$, showing that bortezomib blunts the denervation-induced increase in proteasome activity. RFU, relative fluorescence units. Data are presented as mean \pm SD. Statistical analysis performed with an unpaired, 2-tailed Student's $t$ test in $\mathbf{C}$ and $\mathbf{F}$; and with unpaired, 2-tailed Student's $t$ test between groups and paired, 2-tailed Student's $t$ test between limbs of mice in each group in G-J. Bonferroni corrections for multiple comparisons were performed in $\mathbf{C}$ and $\mathbf{G}-\mathbf{J}$, and adjusted $P$ values are reported for those data. ${ }^{* *} P<0.01,{ }^{* *} P<0.001,{ }^{* * * *} P<0.0001$.

whole muscle length or contribute to contracture pathology. Remarkably, proteasome inhibition during neonatal growth prevents contractures, representing a potentially novel approach to this debilitating and previously unsolved clinical problem. Although the precise mechanisms by which proteasome activity regulates muscle length remain to be determined, the current study links the cellular process of proteasome activity, the physiologic process of longitudinal muscle growth, and the clinical problem of neuromuscular contractures. By connecting these processes, this study thus shifts the paradigm of neuromuscular contractures from a mechanical to a biological realm, provides proof of concept that a biological solution is feasible for neuromuscular contractures, and sheds light on the regulation of muscle growth in a clinically relevant scenario.

The role of myonuclear accretion in adult muscle homeostasis has been explored in recent years, with evidence from MuSC ablation studies suggesting that myonuclear accretion is necessary for normal muscle hypertrophy during overload $(46,54)$ and regeneration following injury $(55)$. However, the role for MuSC-mediated myonuclear accretion in neonatal muscle growth has only been observationally characterized, as ablation of MuSCs in neonatal animals has been complicated by lethality (37). Nonetheless, myonuclear accretion occurs uniquely during neonatal muscle growth (36), during the time frame of contracture development after NBPI. In addition, the myonuclear domain as a function of length remains constant during neonatal growth (36), suggesting a tight coupling of myonuclear accretion and sarcomerogenesis. Because of these findings, we initially hypothesized that impaired myonuclear accretion would underlie contracture pathogenesis. We were surprised to find that reduction in myonuclear number through genetic deletion of Mymk in progenitors does not impair longitudinal muscle growth or cause contractures. Moreover, we found that the myonuclear domain as a function of length, measured in serial sarcomeres, is able to increase substantially in the absence of normal myonuclear numbers. These data indicate that dysregulation of the final function of MuSCs, to fuse and contribute a new nucleus to the myofiber, cannot be a major mechanism for impaired longitudinal growth and contracture pathogenesis. However, we did observe dysregulation of MuSCs in terms of increased numbers and proliferative ability, potentially suggesting they may respond to or indirectly impact pathogenesis, perhaps through crosstalk with other progenitor populations in muscle (56).

On the surface, our results suggest the pathways that control longitudinal muscle growth in the neonatal period are similar to what leads to atrophy in adult denervated muscle (57). Indeed, we observed increased levels of MuRF1 and elevated 20S proteasome activity. Moreover, we also found elevated protein synthesis in NBPI muscle, consistent with adult denervation-induced atrophy (58). Given these similarities between neonatal and adult denervation, it is surprising that proteasome inhibition was able to completely prevent the contracture phenotype in our model, in contrast with only partial and inconsistent rescue of the loss of muscle mass in adult models of denervation-induced atrophy (53). One difference between neonatal denervation and adult denervation is that myonuclear accretion is occurring in the former condition, and could explain the possibly compensatory activation of protein synthesis. Another difference is that denervation of adult muscle is mainly characterized by atrophy in width of myofibers, whereas neonatal neuromuscular contractures are due to reduced longitudinal growth. Indeed, our data indicate that contracture prevention is accompanied by nearly complete rescue of muscle length without significant rescue of cross-sectional area or volume, suggesting that failure of proteasome inhibition to completely rescue muscle mass does not preclude effective rescue of length. This finding also highlights potentially discordant regulation of muscle length and cross-sectional area, which, together with the discordant effects of myonuclear number on fiber widths between different muscles, warrants further study that is beyond the scope of the current investigation regarding muscle length and contractures. Nonetheless, the path to an effective treatment for neonatal neuromuscular contractures following NBPI may be more straightforward than mitigating adult muscle atrophy in cross-sectional area and mass, as longitudinal growth may be more tightly controlled by protein degradation.

It is important to note that while bortezomib is currently in use for adult cancer treatment and is in clinical trials in children (51), it is associated with toxicity. We minimized toxicity in our model by adjusting the dose and timing of treatment and by coadministering [Gly14]-HN (52), and our optimized dosage 
had minimal or no mortality and no effect on body weight or bone growth. Nonetheless, toxicity remains a concern when attempting to translate these results into the clinic. Further defining the necessary treatment window required to prevent contractures could limit cumulative toxicity from long-term administration. Indeed, denervation outside the neonatal period does not cause contractures (18), suggesting that a finite window of bortezomib treatment may be sufficient. Furthermore, newer-generation proteasome inhibitors have been developed, with more favorable toxicity profiles (59). Finally, exploring the complex regulatory network governing protein dynamics may yield additional targets to restore anabolic proteostasis specifically in neonatally denervated muscle, since systemically administered bortezomib, as was used in this study, is not muscle specific. However, in the current study, bortezomib blunted the denervation-induced increase in muscle proteasome activity, rescued muscle length without altering underlying bone length, and relieved contractures that we have previously demonstrated to be an intrinsic problem of denervated muscles. These data are therefore consistent with bortezomib acting through amelioration of denervated muscle pathology, rather than through other non-muscle effects, even though the precise role and mechanisms of proteasome function in longitudinal muscle growth remain to be determined. Nonetheless, even if bortezomib is not the ultimate therapy used in humans, our findings here provide the proof of concept that proteasome inhibition is sufficient to prevent contractures following NBPI, representing what we believe is the first biological solution to the mechanical problem of neuromuscular contractures.

There are multiple mechanisms by which proteasome inhibition could ameliorate contractures. A likely explanation is that the proteasome could be controlling the contracture phenotype by either degrading signaling proteins that regulate sarcomerogenesis or by degrading sarcomeric proteins themselves. Alternatively, bortezomib could be mediating an effect indirectly though MuSC activity. A limitation of our study is that these mechanisms were not elucidated, but the potential novelty of our current findings lies not in the mechanism of action of bortezomib, but rather in (a) the proof of concept that neuromuscular contractures are a biological rather than purely mechanical problem, and (b) that neonatal longitudinal muscle growth and contractures are mediated by proteostasis rather than myonuclear accretion. These discoveries stimulate many exciting questions regarding the biological mechanisms by which innervation, myonuclear number, proteostasis, and muscle growth are all related. Nonetheless, the newfound relevance of the proteasome to longitudinal muscle growth and neuromuscular contractures opens a new direction of biological investigation with direct translational implications for a previously unsolved clinical problem.

The findings of this study also provide a foundation to develop strategies for preventing contractures in other neuromuscular disorders. Contractures in CP are similarly characterized by impaired longitudinal muscle growth, indicated by sarcomere elongation identical to that seen in our model following NBPI. Although the neurologic pathology differs between NBPI and CP, the perinatal age of onset is similar. Similarly, muscle contractures occur following other early childhood neuromuscular disorders, such as spinal muscular atrophy (60), especially the types with perinatal onset. Therefore, although the relationships between innervation and proteostasis in the neonatal period are not fully elucidated in NBPI or CP, future studies confirming the efficacy of proteasome inhibition in animal models and clinical pediatric populations could ultimately render obsolete the destructive surgeries currently required to alleviate a wide variety of disabling neuromuscular contractures and the secondary skeletal deformities that result from them.

\section{Methods}

NBPI surgical model. Unilateral global (C5-T1) NBPIs were created by surgical extraforaminal nerve root excision in 5-day-old CD-1 mice (Charles River) under general anesthesia. Deficits in motor function were validated postoperatively and again prior to sacrifice to ensure only animals with permanent motor deficits were included for analysis, eliminating potential confounding effects of reinnervation and thus increased limb movement. Mice were allowed activity ad libitum until sacrifice, with no observed deficits in feeding, general mobility, or overall activity. Elbow and shoulder (where indicated) range of motion were measured immediately after sacrifice using a validated digital photography technique in order to confirm the presence of elbow flexion and shoulder internal rotation contractures (16). Mice were euthanized by $\mathrm{CO}_{2}$ asphyxiation, except at the P5 and P12 time points, where isoflurane overdose was utilized.

Immunohistochemistry. Bilateral biceps muscles were harvested, fixed in $10 \%$ neutral buffered formalin (NBF) for 1 hour, and then cryoprotected in sucrose prior to snap freezing in optimum cutting temperature $(\mathrm{OCT})$. Frozen sections $(10 \mu \mathrm{m})$ were taken from the mid-muscle belly region and treated with $10 \mathrm{mM}$ sodium citrate, $\mathrm{pH} 6.0$ heat-mediated antigen retrieval in a rice steamer for 5 minutes. Slides were permeabilized in 
$0.4 \%$ Triton X-100/PBS for 10 minutes and blocked in 10\% normal donkey serum (NDS) (Jackson ImmunoResearch) and $1 \%$ bovine serum albumin (BSA), and then blocked in donkey anti-mouse IgG Fab fragment (1:50, Jackson ImmunoResearch) (with 1\% NDS and 1\% BSA) in PBS for 2 hours each. Primary antibodies were mouse anti-Pax7 (1:100, sc-81648, Santa Cruz Biotechnology) and rabbit anti-MyoD (1:50, sc-760, Santa Cruz Biotechnology), in PBS containing 1\% NDS and 1\% BSA and incubated overnight at $4^{\circ} \mathrm{C}$. Secondary antibodies were donkey anti-mouse IgG-DyLight 549 (1:800, 715-505-150, Jackson ImmunoResearch) and donkey anti-rabbit IgG-DyLight 649 (1:800, 711-495-152, Jackson ImmunoResearch), diluted in PBS containing $1 \% \mathrm{NDS}, 1 \% \mathrm{BSA}$, and $1 \mu \mathrm{g} / \mathrm{mL}$ 4',6-diamidino-2-phenylindole dihydrochloride (DAPI) (Sigma-Aldrich) and incubated for at least 1 hour. Slides were mounted in Vectashield antifade mounting medium (Vector Laboratories) and imaged by widefield epifluorescence on an Axioplan 2 imaging microscope with the Plan Apochromat 20× objective using AxioVision software (Carl Zeiss Microscopy). Three images per muscle sample from 4 mice were analyzed using Imaris software (Bitplane).

CD-1 mice were given BrdU (00-0103, Invitrogen) by daily intraperitoneal (i.p.) injections (10 $\mu \mathrm{L} / \mathrm{g}$ body weight) starting on post-NBPI day 1. At 2 weeks after NBPI (24 hours following the last BrdU injection), bilateral biceps muscles were harvested and snap frozen in OCT. Frozen sections $(10 \mu \mathrm{m})$ were taken from the mid-muscle belly region, fixed in 4\% paraformaldehyde (PFA) in PBS for 5 minutes and treated with $2 \mathrm{~N} \mathrm{HCl}, \mathrm{pH} 0.6-0.9$ for 10 minutes, permeabilized in 0.5\% Triton X-100/PBS for 6 minutes, and blocked as described above. Primary antibodies were mouse anti-Pax7, rat anti-BrdU (1:200, ab6326, Abcam), and rabbit anti-dystrophin (1:250, ab15277, Abcam), diluted in PBS containing 1\% NDS and 1\% BSA and incubated overnight at $4^{\circ} \mathrm{C}$. Secondary antibodies were donkey anti-mouse IgG-Alexa Fluor 555 (1:800, A-31570, Invitrogen), donkey anti-rat-Alexa Fluor 488 (1:800, 712-545-153, Jackson ImmunoResearch), and donkey anti-rabbit-Alexa Fluor 647 (1:800, 711-605-152, Jackson ImmunoResearch), diluted in PBS containing 1\% NDS, $1 \%$ BSA, and $1 \mu \mathrm{g} / \mathrm{mL}$ DAPI, and incubated for at least 1 hour. Slides were mounted in Prolong Gold antifade mountant (Life Technologies) and imaged on a Nikon Eclipse Ti inverted microscope with the Plan Apo VC 20× DIC N2 objective on a Nikon A1R confocal using the $405 \mathrm{~nm}, 488 \mathrm{~nm}, 561 \mathrm{~nm}$, and 638 $\mathrm{nm}$ lasers and NIS-Elements imaging software (Nikon Instruments). Three images ( $\sim 100$ muscle fibers) per muscle sample from 7 mice were analyzed using the Fiji program (61) (https://fiji.sc/; Cell Counter plug-in).

Genetically modified mice. NBPIs were created as described above in 5-day-old Pax TreER Rosa26 ${ }^{\mathrm{LacZ}}$ (double homozygous) transgenic mice (stock numbers 017763 and 009427, The Jackson Laboratory) (62, 63). $\beta$-Galactosidase reporter gene expression was induced in $\mathrm{Pax} 7^{+}$mice with a single dose of tamoxifen $(0.5 \mathrm{mg} / \mathrm{g}$ body weight in corn oil; T5648, Sigma-Aldrich) administered by oral gavage 2 days after NBPI (P7). Bilateral biceps muscles were harvested at 2 weeks after NBPI, snap frozen in OCT, and $10-\mu \mathrm{m}$ frozen sections were taken from the muscle belly region proximal to the shoulder. Sections were then fixed in $2 \%$ PFA/PBS for 5 minutes before using a standard 5-bromo-4-chloro-3-indolyl- $\beta$-D-galactoside (X-Gal) staining protocol with overnight colorimetric development. Slides were mounted in Prolong Gold antifade mountant and imaged on a Nikon 90i microscope with the Plan Apo 20× DIC M objective, Photometrics CoolSNAP HQ2 monochromatic camera, and NIS-Elements imaging software. Color RGB images were generated by setting exposures of the TRITC, GFP, and DAPI filters (with epifluorescence shutters closed) to generate a white background image when merged (manual white-color balance). The colored RGB images were merged and 3 images ( 100 muscle fibers) per muscle sample from 7 mice were analyzed using the Fiji program (Cell Counter plug-in).

$M y m k^{s c K O}$ mice were generated by crossing $M y m k^{\text {loxP/loxP }}$ mice and Pax $7^{\text {CreER }}$ mice to yield Mymk $k^{\text {loxP/loxP }}$ Pax $7_{\text {CreERT2 }}$ mice $(46,47,64)$. These genetically modified alleles are in the C57BL/6 background. Mymk $k^{\text {loxP/loxp }}$ mice served as controls. To delete Mymk in MuSCs, mice were administered $200 \mathrm{mg}$ tamoxifen $(10 \mathrm{mg} / \mathrm{mL}$ in $90 \%$ corn oil $/ 10 \%$ ethanol) by i.p. injection at P0. Muscle was harvested at P5 for expression analysis to confirm downregulation of Mymk. RNA was isolated from the gastrocnemius muscle using TRIzol (Invitrogen), and cDNA was synthesized using MultiScribe reverse transcriptase with random hexamer primers (Applied Biosystems). Gene expression was assessed using PowerUp SYBR Green Master Mix (Applied Biosystems), and performed on a 7900HT fast real-time PCR machine (Applied Biosystems). qPCR was performed using the following primers for Mymk: forward, 5'-ATCGCTACCAAGAGGCGTT-3'; reverse, 5'-CACAGCACAGACAAACCAGG-3'. Results were normalized to glyceraldehyde phosphate dehydrogenase $(\mathrm{GAPDH})$ using the following primers: forward, 5'-TGCGACTTCAACAGCAACTC-3'; reverse, 5'-GCCTCTCTTGCTCAGTGTCC-3'.

To isolate single myofibers, biceps muscles were harvested and incubated in high-glucose DMEM (Hyclone Laboratories) containing $0.2 \%$ collagenase type I (Sigma-Aldrich) at $37^{\circ} \mathrm{C}$ for $45-60$ minutes. 
After 40 minutes of incubation, muscles were gently triturated to loosen the digesting myofibers, and then returned to the incubator for up to 60 total minutes. After incubation, muscles were removed from the $0.2 \%$ collagenase/DMEM solution and placed into PBS. To isolate single myofibers, muscles were triturated using pipettes with bores of decreasing sizes until myofibers shed from the muscle. Single myofibers were collected and fixed in 4\% PFA/PBS for 20-30 minutes at room temperature, and subsequently stored in $\mathrm{PBS}$ at $4^{\circ} \mathrm{C}$. To analyze the number of myonuclei, myofibers were permeabilized in $0.2 \%$ Triton X-100/ PBS for 10 minutes at room temperature, washed 3 times in PBS, and mounted on slides with VectaShield containing DAPI (Vector Laboratories). Myofibers were imaged using a Nikon SpectraX widefield microscope with the $10 \times$ objective. Myonuclei were counted in 3D-reconstructed images using Imaris software (Bitplane). Fifteen to twenty myofibers were analyzed per mouse in each muscle.

Sarcomere lengths were measured from single muscle fibers, acquiring 6 images per fiber by differential interference contrast (DIC) microscopy on a Nikon Eclipse Ti-E inverted microscope with the Plan Apo $\lambda$ $40 \times$ objective (Nikon Instruments), Xyla 4.2 megapixel, 16-bit sCMOS monochromatic camera (Andor/ Oxford Instruments) and NIS-Elements imaging software (Nikon Instruments). A series of 10 sarcomeres were measured per image in AxioVision software and an average sarcomere length was then determined for each fiber. Myofiber widths (diameters) were similarly measured to determine cross-sectional area and volume for myonuclear domain calculations.

Mouse limbs harvested 4 weeks after NBPI were processed on cork at $90^{\circ}$ elbow flexion (confirmed by digital x-ray, which was also used to measure bone length and assess for any bone deformity) prior to fixation in $10 \% \mathrm{NBF}$ as described previously (16). Brachialis muscles were then removed, digested in $15 \%$ sulfuric acid for 30 minutes to obtain muscle bundles (16), and imaged for sarcomere length measurement by DIC microscopy as described above.

Gene expression analysis of NBPI muscle. Total RNA was extracted from snap-frozen bilateral biceps muscles from 3 mice harvested 3 weeks after NBPI using the ReliaPrep RNA tissue miniprep system (Promega). The concentration and quality of the RNA samples were determined using the Bioanalyzer (Agilent), and 10 ng of each amplified using the Ovation RNA-Seq system V2 (NuGEN), constructed into cDNA libraries using the Nextera XT DNA sample preparation kit (Illumina), and sequenced on the HiSeq 2500 system (Illumina, paired-end 75-bp flow cell) to a depth of at least 35-40 million reads. Resulting FASTQ sequences were pseudoaligned against the Mus musculus transcriptome (EnsMart72/mm10) using the Kallisto (65) program and analyzed with the AltAnalyze (66) program (transcripts per million [TPM] filtered by adjP value and 2-fold change in gene expression). Gene ontology analysis was performed on the genes that changed by $\log _{2}$ fold change to select for genes that exhibited the most robust differential regulation. Here, 336 genes were upregulated and 21 genes were downregulated. The 336 upregulated genes were analyzed for enrichment of biological processes using the Gene Ontology Consortium (http://www.geneontology. org) $(67,68)$. The RNA sequencing data in this publication have been deposited in NCBI's Gene Expression Omnibus (69) and are available through accession number GSE137606.

To assess MuRF1 transcript levels, RNA was extracted from snap-frozen bilateral biceps muscles from 6 mice harvested 2 weeks after NBPI as described above, and 500 ng of each was used in first-strand cDNA synthesis using the GoScript reverse transcription system (Promega) with both oligo(dT)15 and random primers $\left(0.5 \mu \mathrm{g}\right.$ each primer/reaction) carried out for 1 hour at $50^{\circ} \mathrm{C}$, followed by heat inactivation. Primers for PCR were designed using the Primer3 $(70,71)$ program (http://bioinfo.ut.ee/primer3/) so that 1 primer per set bound across an exon-exon boundary. MuRF1 (Trim63) gene target transcript Trim63-202 (ENSMUST00000105875.7) forward, 5'-GGAGAACCTGGAGAAGCAGC-3' and reverse, 5'-TAGGGATTCGCAGCCTGGAA-3'; and Atp5j gene normalizer transcript Atp5j-201 (ENSMUST00000023608.13) forward, 5'-TCAGTGCAAGTACAGAGACTCA-3' and reverse, 5'-GCCTGTCGCTTTGATTTGTACT-3'. The gene Atp5j (ENSMUSG00000022890) was chosen for normalization due to finding that it was expressed at similar high levels between 3-week post-NBPI and contralateral control biceps muscles in the RNA sequencing data.

Each $20 \mu \mathrm{L}$ PCR contained $1 \times$ GoTaq qPCR master mix (Promega; containing a proprietary dye detected with the SYBR channel), $0.2 \mu \mathrm{L}$ CXR reference dye (detected with the ROX channel), 5 pmol each primer and $2 \mu \mathrm{L}$ cDNA (diluted 1:10); and was carried out in a 96-well plate on the StepOnePlus real-time PCR system (Applied Biosystems). PCR cycling consisted of hot-start activation at $95^{\circ} \mathrm{C}$ for 2 minutes, 40 cycles of denaturation at $95^{\circ} \mathrm{C}$ for 15 seconds, annealing at $54^{\circ} \mathrm{C}$ (Atp5j) or $56^{\circ} \mathrm{C}$ (Trim63) for 15 seconds and extension at $60^{\circ} \mathrm{C}$ for 1 minute (data acquisition at the end of step to measure rate of amplification); final dissociation for 1 cycle at $95^{\circ} \mathrm{C}$ for 15 seconds, and then melt curve analysis starting 
at $60^{\circ} \mathrm{C}$ for 1 minute and then $+0.3^{\circ} \mathrm{C}$ for 15 seconds per temperature interval until $95^{\circ} \mathrm{C}$ with continuous data acquisition to confirm the generation of a single PCR product. Average $C_{t}$ was determined from triplicate reactions using the StepOne software (Applied Biosystems) and fold difference in gene expression determined using the Comparative $C_{t}\left(\Delta \Delta C_{t}\right)$ method, with correction of PCR efficiency $(E=$ $10^{[-1 / \text { slope }]}$ ) between target (Trim63) and normalizer (Atp5j) primer sets determined from a 4-point standard curve $(1: 5,1: 50,1: 500$, and 1:5000 dilutions of pooled cDNA from test bilateral biceps muscles from 1 mouse harvested on week 3 after NBPI prepared as described above) included in each primer set reaction run, using the following equations (72): $\Delta \mathrm{C}_{\mathrm{t} \text {-target }}=\mathrm{C}_{\mathrm{t} \text {-GOI }}{ }^{\mathrm{c}}-\mathrm{C}_{\mathrm{t} \text {-GOI }}{ }^{\mathrm{s}}, \Delta \mathrm{C}_{\mathrm{t} \text {-normalizer }}=\mathrm{C}_{\mathrm{t} \text {-norm }}{ }^{\mathrm{c}}-\mathrm{C}_{\mathrm{t} \text {-norm }}{ }^{\mathrm{s}}$, and fold difference $=\left(\mathrm{E}_{\text {target }}\right)^{\Delta \mathrm{C} \text { t-target }} /\left(\mathrm{E}_{\text {normalizer }}\right)^{\Delta \mathrm{C} \text {-normalizer }}$, where $s$ represents individual mouse samples from bilateral biceps at 2 weeks after NBPI (6 mice), and $c$ represents the calibrator sample derived from unilateral biceps from unoperated mice that are age-matched to 3 weeks after NBPI (average of 3 mice).

Analysis of protein dynamics after NBPI. NBPIs were created as described above in 5-day-old mice. Surface sensing of translation (SUnSET) $(73,74)$ was performed by administration of puromycin $(21.8$ $\mathrm{mg} / \mathrm{kg}$ body weight; P7255, Sigma-Aldrich) by i.p. injection 30 minutes prior to sacrifice at weekly time points, beginning immediately postoperatively until 4 weeks after NBPI. Total proteins were extracted from snap-frozen bilateral biceps muscles using radioimmunoprecipitation assay (RIPA) buffer containing cOmplete ULTRA protease inhibitor cocktail (Roche) and PhosSTOP phosphatase inhibitor cocktail (Roche) and centrifuged at $20,000 \mathrm{~g}$ for 20 minutes at $4^{\circ} \mathrm{C}$. Proteins were then precipitated with acetone and resuspended in $1 \times$ Laemmli sample buffer (161-0737, Bio-Rad) prepared with 2-mercaptoethanol and RIPA buffer, and heat denatured for 5 minutes at $95^{\circ} \mathrm{C}$. Equal protein loads were run in $4 \%-15 \%$ Mini-PROTEAN TGX gels (456-1086, Bio-Rad) in $25 \mathrm{mM}$ Tris, $192 \mathrm{mM}$ glycine, 0.1\% SDS running buffer, and transferred to Immobilon-FL polyvinylidene fluoride (PVDF) (IPFL10100, Millipore) in transfer buffer consisting of $25 \mathrm{mM}$ Tris, $192 \mathrm{mM}$ glycine, and 20\% methanol. Western blot analysis was carried out using the following antibodies: rat anti-puromycin (1:1000, MABE341, Sigma-Aldrich) and rabbit anti-K48-linkage-specific polyubiquitin (1:1000, 8081S, Cell Signaling Technology). Western blots were detected using species-specific secondary antibodies raised in donkey and conjugated to either Alexa Fluor 680 or 790 (1:100,000, Jackson ImmunoResearch) imaging with the Odyssey CLx, and signal intensities measured using the Image Studio Lite program (LI-COR Biosciences). Western blot signals were normalized to gel protein load.

To assay proteasome activity, bilateral biceps muscles from 6 mice harvested 2 weeks after NBPI were snap frozen, extracted in 20 mM Tris-HCl, pH 7.2, 0.1 mM EDTA, 1 mM 2-mercaptoethanol, 5 mM ATP, 20\% glycerol, $0.04 \%$ Nonidet P-40 and centrifuged at $13,000 \mathrm{~g}$ for 15 minutes at $4^{\circ} \mathrm{C}$ (75). Protein concentration was determined using the Pierce $660 \mathrm{~nm}$ protein assay kit (Thermo Fisher Scientific) and $25 \mu \mathrm{g}$ total protein per muscle used to assay the chymotrypsin-like activity of the $20 \mathrm{~S}$ proteasome $\beta-5$ catalytic subunit through detection of 7-amino-4-methylcoumarin (AMC) fluorescence by cleavage of the peptide substrate Suc-LLVY-AMC (S-280, Boston Biochem) in 25 mM HEPES, pH 7.5, 0.5 mM EDTA, 0.05\% NP-40, and 0.001\% SDS. Assay design was based on the Chemicon kit (APT280) and duplicate reactions were carried out in a white opaque polystyrene 96-well plate for 2 hours at $37^{\circ} \mathrm{C}$, with endpoint fluorescence measured at $380 \mathrm{~nm} / 460 \mathrm{~nm}$ in a SpectraMax M5 microplate reader (Molecular Devices). Relative fluorescence units were then calculated per $\mu$ g protein.

Bortezomib treatment. Mice were treated either with saline (as the vehicle; 0.9\% Sodium Chloride Injection USP, Hospira), [Gly14]-HN (1 $\mu \mathrm{g}$ /dose; H6161, Sigma-Aldrich) alone or coadministered with bortezomib (0.2-0.4 mg/kg body weight; 5043140001, Sigma-Aldrich) by i.p. injection starting immediately postoperatively or delayed by 3 or 7 days (P8 or 12 start), and injected every other day with sacrifice at 4 weeks after NBPI (24 hours following the last i.p. injection). The use of littermate controls was rejected due to the risk of treatment cross-contamination either through direct contact or by ingestion from their mother, and [Gly14]$\mathrm{HN}$ was included to mitigate the toxicity that has been reported for bortezomib (52). Deficits in motor function were confirmed as described above, and measurement of shoulder and elbow range of motion was measured 4 weeks after NBPI immediately after sacrifice (16) with blinding to the treatment group. Bilateral biceps muscles were harvested and processed as for the Chemicon proteasome activity assay as described above. The remaining forelimbs were harvested and positioned on cork at $90^{\circ}$ elbow flexion (confirmed by digital x-ray) prior to fixation in $10 \% \mathrm{NBF}$ as described previously (16). Brachialis muscles were then removed, soaked in 25\% Lugol solution (32922, Sigma-Aldrich) in PBS overnight, and imaged by micro computed tomography (microCT) (Inveon, Siemens) at $20-\mu \mathrm{m}$ resolution. Volume rendering of digital imaging and communications in medicine (DICOM) images was performed using the Fiji program (Segmentation Editor and 3D Viewer plug-ins) to obtain muscle volume and cross-sectional area (76). Brachialis muscles were then recovered by 
soaking in PBS overnight at $4^{\circ} \mathrm{C}$, digestion in $15 \%$ sulfuric acid for 30 minutes to obtain muscle bundles (16), and imaged for sarcomere length measurement by DIC microscopy as described above. Humerus lengths were measured using AxioVision on the digital x-rays used to confirm symmetric elbow flexion positions during fixation, measuring from the proximal humerus physis to the distal articular surface to exclude the potential confounding effect of variable ossification of the humeral head following denervation.

Statistics. For all continuous data, outliers were detected a priori by Grubbs's test and excluded. All continuous data with $n>3$ animals were tested for normality with the Shapiro-Wilk test. Normally distributed data and data with $n=3$ were compared with a 2-tailed Student's $t$ test, paired where parameters were compared between forelimbs (NBPI versus contralateral) in individual animals, and unpaired when parameters were compared between animals. Non-normally distributed data were compared using Mann-Whitney $U$ tests for unpaired data or Wilcoxon's signed-rank tests for paired analyses where parameters were compared between forelimbs (NBPI versus contralateral). Based on the above rules regarding pairing and normality, we selected specific statistical tests for each comparison within certain experiments, rather than using ANOVA to detect differences among multiple groups. Bonferroni corrections for multiple comparisons were used to for any such comparisons across multiple groups, with adjusted $P$ values reported where indicated. All data are presented as mean $\pm \mathrm{SD}$. The degree of significance between data sets is depicted as follows: ${ }^{*} P<0.05,{ }^{* *} P<0.01,{ }^{* * *} P<0.001,{ }^{* * *} P<0.0001$. A priori power analyses based on prior work were performed for the phenotypic variables of contracture severity, determining that 6 mice per group were required for at least $80 \%$ power to detect a $10^{\circ}$ difference in contractures and a $0.2-\mu \mathrm{m}$ difference in sarcomere lengths between experimental conditions.

Study approval. All animal procedures were approved by Cincinnati Children's Hospital Medical Center's Institutional Animal Care and Use Committee.

\section{Author contributions}

RC and DPM conceived the study. SN, AAWC, LH, QG, and RC performed experiments. SN, AAWC, LH, QG, DPM, and RC analyzed data. RC, DPM, and SN wrote the manuscript with assistance from all authors.

\section{Acknowledgments}

We thank the following people and core facilities at Cincinnati Children's Hospital Medical Center: Kritton Shay-Winkler for additional technical assistance; Jenny Melzer from Veterinary Services; Matt Kofron from the Confocal Imaging Core; Shawn Smith, Hung Chi Liang, and Kelly Rangel from the Gene Expression Core; Sharon Wang from the Preclinical Imaging Core (University of Cincinnati College of Medicine); and Benjamin Liou for kindly providing use of their plate reader. This work was supported by grants to DPM from the NIH (R01AR068286, R01AG059605) and the Pew Charitable Trusts. Work in the Cornwall laboratory was supported by a Career Development Grant to RC from the Orthopaedic Research and Education Foundation, as well as funding from the Cincinnati Children's Hospital Division of Orthopaedic Surgery and Junior Cooperative Society.

Address correspondence to: Roger Cornwall, Cincinnati Children's Hospital Medical Center, 3333 Burnet Avenue, MLC 2017, Cincinnati, Ohio 45208, USA. Phone: 513.636.7319; Email: roger.cornwall@cchmc.org. Or to: Douglas Millay, Cincinnati Children's Hospital Research Foundation, 240 Albert Sabin Way, MLC 7020, Cincinnati, Ohio 45208, USA. Phone: 513.803.7437; Email: douglas.millay@cchmc.org.

1. Cornwall R, Waters PM. Pediatric brachial plexus palsy. In: Wolfe SW, Hotchkiss RN, Pederson WC, Kozin SK, Cohen MS, eds. Green's Operative Hand Surgery. Philadelphia, PA: Elsevier; 2017:1391-1424.

2. Yeargin-Allsopp M, Van Naarden Braun K, Doernberg NS, Benedict RE, Kirby RS, Durkin MS. Prevalence of cerebral palsy in 8-year-old children in three areas of the United States in 2002: a multisite collaboration. Pediatrics. 2008;121(3):547-554.

3. Foad SL, Mehlman CT, Ying J. The epidemiology of neonatal brachial plexus palsy in the United States. J Bone Joint Surg Am. 2008;90(6):1258-1264.

4. Graham HK, et al. Cerebral palsy. Nat Rev Dis Primers. 2016;2:15082.

5. Waters PM, Smith GR, Jaramillo D. Glenohumeral deformity secondary to brachial plexus birth palsy. J Bone Joint Surg Am. 1998;80(5):668-677.

6. Sibinski M, Woźniakowski B, Drobniewski M, Synder M. Secondary gleno-humeral joint dysplasia in children with persistent obstetric brachial plexus palsy. Int Orthop. 2010;34(6):863-867.

7. Kozin SH. Correlation between external rotation of the glenohumeral joint and deformity after brachial plexus birth palsy. $J$ Pediatr 
Orthop. 2004;24(2):189-193.

8. Larnert P, Risto O, Hägglund G, Wagner P. Hip displacement in relation to age and gross motor function in children with cerebral palsy. J Child Orthop. 2014;8(2):129-134.

9. Miller F, Cardoso Dias R, Dabney KW, Lipton GE, Triana M. Soft-tissue release for spastic hip subluxation in cerebral palsy. $J$ Pediatr Orthop. 1997;17(5):571-584.

10. Waters PM. Update on management of pediatric brachial plexus palsy. J Pediatr Orthop. 2005;25(1):116-126.

11. Quinby JM, Abraham A. Musculoskeletal problems in cerebral palsy. Curr Paediatr. 2005;15(1):9-14.

12. Newman CJ, Morrison L, Lynch B, Hynes D. Outcome of subscapularis muscle release for shoulder contracture secondary to brachial plexus palsy at birth. J Pediatr Orthop. 2006;26(5):647-651.

13. Tilton AH. Therapeutic interventions for tone abnormalities in cerebral palsy. NeuroRx. 2006;3(2):217-224.

14. van der Sluijs JA, van Ouwerkerk WJ, de Gast A, Nollet F, Winters H, Wuisman PI. Treatment of internal rotation contracture of the shoulder in obstetric brachial plexus lesions by subscapular tendon lengthening and open reduction: early results and complications. J Pediatr Orthop B. 2004;13(3):218-224.

15. Orendurff MS, Aiona MD, Dorociak RD, Pierce RA. Length and force of the gastrocnemius and soleus during gait following tendo Achilles lengthenings in children with equinus. Gait Posture. 2002;15(2):130-135.

16. Nikolaou S, Peterson E, Kim A, Wylie C, Cornwall R. Impaired growth of denervated muscle contributes to contracture formation following neonatal brachial plexus injury. J Bone Joint Surg Am. 2011;93(5):461-470.

17. Cohen MS, Schimmel DR, Masuda K, Hastings H, Muehleman C. Structural and biochemical evaluation of the elbow capsule after trauma. J Shoulder Elbow Surg. 2007;16(4):484-490.

18. Weekley H, Nikolaou S, Hu L, Eismann E, Wylie C, Cornwall R. The effects of denervation, reinnervation, and muscle imbalance on functional muscle length and elbow flexion contracture following neonatal brachial plexus injury. J Orthop Res. 2012;30(8):1335-1342.

19. Hogendoorn S, van Overvest KL, Watt I, Duijsens AH, Nelissen RG. Structural changes in muscle and glenohumeral joint deformity in neonatal brachial plexus palsy. J Bone Joint Surg Am. 2010;92(4):935-942.

20. Foran JR, Steinman S, Barash I, Chambers HG, Lieber RL. Structural and mechanical alterations in spastic skeletal muscle. Dev Med Child Neurol. 2005;47(10):713-717.

21. Nikolaou S, et al. Contribution of denervated muscle to contractures after neonatal brachial plexus injury: not just muscle fibrosis. Muscle Nerve. 2014;49(3):398-404.

22. Felder A, Ward SR, Lieber RL. Sarcomere length measurement permits high resolution normalization of muscle fiber length in architectural studies. J Exp Biol. 2005;208(Pt 17):3275-3279.

23. Crouch DL, et al. Biomechanical basis of shoulder osseous deformity and contracture in a rat model of brachial plexus birth palsy. J Bone Joint Surg Am. 2015;97(15):1264-1271.

24. Mascarenhas VV, et al. The role of subscapularis muscle denervation in the pathogenesis of shoulder internal rotation contracture after neonatal brachial plexus palsy: a study in a rat model. J Orthop Res. 2014;32(12):1675-1679.

25. Eismann EA, Little KJ, Laor T, Cornwall R. Glenohumeral abduction contracture in children with unresolved neonatal brachia plexus palsy. J Bone Joint Surg Am. 2015;97(2):112-118.

26. Russo SA, et al. Scapulothoracic and glenohumeral contributions to motion in children with brachial plexus birth palsy. $J$ Shoulder Elbow Surg. 2014;23(3):327-338.

27. Russo SA, Loeffler BJ, Zlotolow DA, Kozin SH, Richards JG, Ashworth S. Limited glenohumeral cross-body adduction in children with brachial plexus birth palsy: a contributor to scapular winging. J Pediatr Orthop. 2015;35(3):240-245.

28. Cheng W, Cornwall R, Crouch DL, Li Z, Saul KR. Contributions of muscle imbalance and impaired growth to postural and osseous shoulder deformity following brachial plexus birth palsy: a computational simulation analysis. J Hand Surg Am. 2015;40(6):1170-1176.

29. Crouch DL, Plate JF, Li Z, Saul KR. Computational sensitivity analysis to identify muscles that can mechanically contribute to shoulder deformity following brachial plexus birth palsy. J Hand Surg Am. 2014;39(2):303-311.

30. Fridén J, Lieber RL. Spastic muscle cells are shorter and stiffer than normal cells. Muscle Nerve. 2003;27(2):157-164.

31. Kadam SD, Mulholland JD, Smith DR, Johnston MV, Comi AM. Chronic brain injury and behavioral impairments in a mouse model of term neonatal strokes. Behav Brain Res. 2009;197(1):77-83.

32. Lubics A, et al. Neurological reflexes and early motor behavior in rats subjected to neonatal hypoxic-ischemic injury. Behav Brain Res. 2005;157(1):157-165.

33. Wright J, Rang M. The spastic mouse. And the search for an animal model of spasticity in human beings. Clin Orthop Relat Res. 1990;(253):12-19.

34. Ho ES. Evaluation of pediatric upper extremity peripheral nerve injuries. J Hand Ther. 2015;28(2):135-142.

35. Dumont NA, Bentzinger CF, Sincennes MC, Rudnicki MA. Satellite cells and skeletal muscle regeneration. Compr Physiol. 2015;5(3):1027-1059.

36. White RB, Biérinx AS, Gnocchi VF, Zammit PS. Dynamics of muscle fibre growth during postnatal mouse development. BMC Dev Biol. 2010;10:21.

37. Pallafacchina G, Blaauw B, Schiaffino S. Role of satellite cells in muscle growth and maintenance of muscle mass. Nutr Metab Cardiovasc Dis. 2013;23 Suppl 1:S12-S18.

38. Rodrigues Ade C, Schmalbruch H. Satellite cells and myonuclei in long-term denervated rat muscles. Anat Rec. $1995 ; 243(4): 430-437$.

39. Dayanidhi S, Dykstra PB, Lyubasyuk V, McKay BR, Chambers HG, Lieber RL. Reduced satellite cell number in situ in muscular contractures from children with cerebral palsy. J Orthop Res. 2015;33(7):1039-1045.

40. Kinney MC, Dayanidhi S, Dykstra PB, McCarthy JJ, Peterson CA, Lieber RL. Reduced skeletal muscle satellite cell number alters muscle morphology after chronic stretch but allows limited serial sarcomere addition. Muscle Nerve. 2017;55(3):384-392.

41. Mathewson MA, Lieber RL. Pathophysiology of muscle contractures in cerebral palsy. Phys Med Rehabil Clin N Am. 2015;26(1):57-67.

42. Smith LR, Chambers HG, Lieber RL. Reduced satellite cell population may lead to contractures in children with cerebral palsy. Dev Med Child Neurol. 2013;55(3):264-270. 
43. Rodrigues Ade C, Geuna S, Rodrigues SP, Silva MD, Aragon FF. Satellite cells and myonuclei in neonatally denervated rat muscle. Ital J Anat Embryol. 2002;107(1):51-56.

44. Domenighetti AA, et al. Loss of myogenic potential and fusion capacity of muscle stem cells isolated from contractured muscle in children with cerebral palsy. Am J Physiol, Cell Physiol. 2018;315(2):C247-C257.

45. Millay DP, et al. Myomaker is a membrane activator of myoblast fusion and muscle formation. Nature. 2013;499(7458):301-305.

46. Goh Q, Millay DP. Requirement of myomaker-mediated stem cell fusion for skeletal muscle hypertrophy. Elife. $2017 ; 6:$ e20007.

47. Millay DP, Sutherland LB, Bassel-Duby R, Olson EN. Myomaker is essential for muscle regeneration. Genes Dev. 2014;28(15):1641-1646.

48. Furuno K, Goodman MN, Goldberg AL. Role of different proteolytic systems in the degradation of muscle proteins during denervation atrophy. J Biol Chem. 1990;265(15):8550-8557.

49. Bodine SC, Baehr LM. Skeletal muscle atrophy and the E3 ubiquitin ligases MuRF1 and MAFbx/atrogin-1. Am J Physiol Endocrinol Metab. 2014;307(6):E469-E484.

50. Meng L, Mohan R, Kwok BH, Elofsson M, Sin N, Crews CM. Epoxomicin, a potent and selective proteasome inhibitor, exhibits in vivo antiinflammatory activity. Proc Natl Acad Sci USA. 1999;96(18):10403-10408.

51. Citrin R, Foster JB, Teachey DT. The role of proteasome inhibition in the treatment of malignant and non-malignant hematologic disorders. Expert Rev Hematol. 2016;9(9):873-889.

52. Cohen P. New role for the mitochondrial peptide humanin: protective agent against chemotherapy-induced side effects. $J$ Nat Cancer Inst. 2014;106(3):dju006.

53. Beehler BC, Sleph PG, Benmassaoud L, Grover GJ. Reduction of skeletal muscle atrophy by a proteasome inhibitor in a rat model of denervation. Exp Biol Med (Maywood). 2006;231(3):335-341.

54. Egner IM, Bruusgaard JC, Gundersen K. Satellite cell depletion prevents fiber hypertrophy in skeletal muscle. Development. 2016;143(16):2898-2906.

55. Relaix F, Zammit PS. Satellite cells are essential for skeletal muscle regeneration: the cell on the edge returns centre stage. Development. 2012;139(16):2845-2856

56. Fry CS, Kirby TJ, Kosmac K, McCarthy JJ, Peterson CA. Myogenic progenitor cells control extracellular matrix production by fibroblasts during skeletal muscle hypertrophy. Cell Stem Cell. 2017;20(1):56-69.

57. Sandri M. Protein breakdown in muscle wasting: role of autophagy-lysosome and ubiquitin-proteasome. Int J Biochem Cell Biol. 2013;45(10):2121-2129.

58. Quy PN, Kuma A, Pierre P, Mizushima N. Proteasome-dependent activation of mammalian target of rapamycin complex 1 (mTORC1) is essential for autophagy suppression and muscle remodeling following denervation. J Biol Chem. 2013;288(2):1125-1134

59. Yong K, Gonzalez-McQuire S, Szabo Z, Schoen P, Hajek R. The start of a new wave: Developments in proteasome inhibition in multiple myeloma. Eur J Haematol. 2018;101(2):220-236.

60. Wang HY, Ju YH, Chen SM, Lo SK, Jong YJ. Joint range of motion limitations in children and young adults with spinal muscular atrophy. Arch Phys Med Rehabil. 2004;85(10):1689-1693.

61. Schindelin J, et al. Fiji: an open-source platform for biological-image analysis. Nat Methods. 2012;9(7):676-682.

62. Murphy MM, Lawson JA, Mathew SJ, Hutcheson DA, Kardon G. Satellite cells, connective tissue fibroblasts and their interactions are crucial for muscle regeneration. Development. 2011;138(17):3625-3637.

63. Soriano P. Generalized lacZ expression with the ROSA26 Cre reporter strain. Nat Genet. 1999;21(1):70-71.

64. Lepper C, Conway SJ, Fan CM. Adult satellite cells and embryonic muscle progenitors have distinct genetic requirements. Nature. 2009;460(7255):627-631.

65. Bray NL, Pimentel H, Melsted P, Pachter L. Near-optimal probabilistic RNA-seq quantification. Nat Biotechnol. 2016;34(5):525-527.

66. Salomonis N, et al. Alternative splicing in the differentiation of human embryonic stem cells into cardiac precursors. PLoS Comput Biol. 2009;5(11):e1000553.

67. Ashburner M, et al. Gene ontology: tool for the unification of biology. The Gene Ontology Consortium. Nat Genet. 2000;25(1):25-29.

68. The Gene Ontology Consortium. Expansion of the Gene Ontology knowledgebase and resources. Nucleic Acids Res. 2017;45(D1):D331-D338.

69. Edgar R, Domrachev M, Lash AE. Gene Expression Omnibus: NCBI gene expression and hybridization array data repository. Nucleic Acids Res. 2002;30(1):207-210.

70. Koressaar T, Remm M. Enhancements and modifications of primer design program Primer3. Bioinformatics. 2007;23(10):1289-1291.

71. Untergasser A, et al. Primer3--new capabilities and interfaces. Nucleic Acids Res. 2012;40(15):e115

72. [No authors listed]. Real-time PCR handbook. Thermo Fisher Scientific Inc. https://www.thermofisher.com/content/dam/ LifeTech/global/Forms/PDF/real-time-pcr-handbook.pdf. Accessed October 29, 2019.

73. Schmidt EK, Clavarino G, Ceppi M, Pierre P. SUnSET, a nonradioactive method to monitor protein synthesis. Nat Methods. 2009;6(4):275-277.

74. Goodman CA, et al. Novel insights into the regulation of skeletal muscle protein synthesis as revealed by a new nonradioactive in vivo technique. FASEB J. 2011;25(3):1028-1039.

75. Penna F, et al. Effect of the specific proteasome inhibitor bortezomib on cancer-related muscle wasting. J Cachexia Sarcopenia Muscle. 2016;7(3):345-354

76. Nikolaou S, Hu L, Cornwall R. Afferent innervation, muscle spindles, and contractures following neonatal brachial plexus injury in a mouse model. J Hand Surg Am. 2015;40(10):2007-2016. 